\title{
Polar motions equivalent to high frequency nutations for a nonrigid Earth with anelastic mantle ${ }^{\star}$
}

\author{
P. M. Mathews ${ }^{1}$ and P. Bretagnon ${ }^{2}$ \\ 1 Department of Theoretical Physics, University of Madras, Chennai 600 025, India \\ e-mail: sonny@eth.net \\ 2 IMCCE, Paris Observatoire de Paris, 75014 Paris, France
}

Received 2 September 2002 / Accepted 20 November 2002

\begin{abstract}
The coefficients of polar motions of the rigid/nonrigid Earth in frequency bands other than the retrograde diurnal one are systematically computed using general expressions, derived here for the first time, for the prograde and retrograde torques exerted on the Earth by lunisolar potentials of arbitrary spherical harmonic type. Taken together with the already known coefficients of low frequency nutations and UT1 variations, they provide a complete characterization, with high precision, of the motions of the pole of the terrestrial reference frame in space; this is needed for high precision studies in astronomy and space geodesy. The inputs used for our computations are a table of tidal amplitudes, and values of the geopotential coefficients of degrees up to 4 and of other relevant basic Earth parameters. General relations which connect the coefficients of high frequency nutations to those of the equivalent polar motions are established and used for deducing the former. The Chandler resonance plays a significant role in low frequency polar motions. In this context, the role of mantle anelasticity and the nature of the Earth's deformational response to zero frequency forcing are given special consideration. The free core nutation (FCN) resonance of low frequency nutations is shown to affect the prograde semidiurnal nutations through the coupling produced between the nutations in the two frequency bands by triaxiality terms in the angular momenta of the whole Earth and of its fluid core. It is shown in a transparent fashion that the effect of the core triaxiality arises almost exclusively from the huge FCN-related resonance in the wobble of the core. The magnitude of the effect is found to be a few times smaller than reported in a recent paper; it is also found, unlike in that paper, that the changes in the eigenfrequencies due to trixiality are only of the second order in the triaxiality parameter. Numerical results for the polar motions of the nonrigid Earth in different frequency bands, as well as for the elliptical nutations of the rigid Earth, are tabulated and compared with available numbers from earlier works.
\end{abstract}

Key words. reference systems - Earth

\section{Introduction}

High precision studies in astronomy as well as in certain areas of geophysics and geodesy call for a sufficiently precise model of the motions of the terrestrial reference frame (TRF) in space. The motions of the pole of the TRF relative to that of the celestial reference frame (CRF) are described as nutations or polar motions, depending on the viewpoint. Recent treatments of the nutations of the hypothetical rigid Earth, (e.g., Bretagnon et al. 1997, 1998; Souchay \& Kinoshita 1996, 1997; Souchay et al. 1999; Roosbeek \& Dehant 1998) have aimed at accuracies of 0.1 microarcseconds ( $\mu$ as) or better in the coefficients of nutation. The new nutation series thus constructed include

\footnotetext{
Send offprint requests to: P. M. Mathews, e-mail: sonny@eth.net

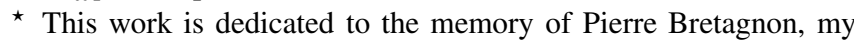
valued colleague and personal friend, and a scientist of great integrity widely respected for his definitive works carried out with meticulous care and precision. He passed away shortly before the final revision of this paper was complete. P. M. M.
}

a considerable number of short period or high frequency (HF) nutations, having frequencies higher than 0.5 cycles per sidereal day (cpsd), and with coefficients up to about $15 \mu$ as in magnitude for $\Delta \psi \sin \epsilon$ and $\Delta \epsilon$. Low frequency nutations are those with frequencies under $0.5 \mathrm{cpsd}$. The nutations referred to here are motions, relative to the CRF, of the figure axis of the Earth with time dependent deformations diregarded. The pole of the figure axis will be taken to coincide with the pole of the TRF, the offset between the two being too small to be of interest for the present purposes.

Conventionally, only the low frequency part (as seen from space) of the relative motion of the poles of the CRF and TRF around each other is viewed as nutation. The remaining part of the motion is pictured as "polar motion" as seen from the terrestrial frame, with a spectrum which includes a low frequency part; in fact, the major components of polar motion are contained in this low frequency part. It is important to take note that the term "polar motion" as employed in this context means a motion of the pole of the celestial frame as seen from the 
terrestrial frame. This specific meaning is implicit in the relationship of polar motion to nutation as presented by Gross (1992) (see also Brzeziński \& Capitaine 1993). (Historically, the motion of the rotation axis around the figure axis - what is called "wobble" in this work - was usually referred to as polar motion. While the two types of usage are nearly equivalent in practical terms in the case of polar motions of very low frequencies (e.g., the Chandler wobble), the two are quite different when the polar motions involved are in high frequency bands. It is necessary to keep the distinction clearly in mind to avoid confusion.)

The 1980 IAU theory of nutation (Seidelmann 1982) envisaged only polar motions of low frequencies, below $0.5 \mathrm{cpsd}$ in magnitude in the TRS, besides low frequency nutations. With the restriction to long periodicities, estimation of the coefficients of spectral components of both types of motions from Very Long Baseline Interferometry (VLBI) data, which are available only at intervals of a few days, became straightforward.

The representation adopted recently by the International Astronomical Union (IAU) for the transformation between the celestial and terrestrial reference frames conforms to the convention of separating the relative motions of the poles of the CRF and the TRF into nutations and polar motions, but with a broadening of the concept of polar motions to include high frequency components too. Insofar as nutation and polar motion are visualized, in the transformation, as motions of the "Celestial Intermediate Pole" (CIP), it needs to be recognized that for those spectral components of the relative motions of the two poles that are of low (high) frequencies in the celestial frame, the CIP is identified, in effect, with the pole of the TRF (CRF).

It is necessary, in view of the above convention, that results relating to what has been referred to in the literature hitherto as "high frequency" or "short period" or "diurnal and subdiurnal" nutation be expressed now in the alternative language of polar motion (PM) with frequencies lying outside the retrograde diurnal band. The presentation of the numerical results in this paper will be done accordingly. We shall, however, use both the alternative terminologies at will, and shall indicate how one may deduce simply, from the results for polar motions, the corresponding results for HF nutations for comparison with the results of earlier authors.

Each spectral component of nutation or polar motion is associated with a corresponding wobble. Wobbles are motions of the rotation axis of the mantle or of either of the core regions relative to a terrestrial frame; where a particular region is not specifically referred to, it is to be understood that the wobble of the mantle is meant.

The frequency of a spectral component of the forced wobble motion is the same as that of the torque which excites the wobble; so is the frequency of the associated polar motion. The frequency of the corresponding nutation, being relative to a space-fixed frame, is higher by $1 \mathrm{cpsd}$ (the mean rate of Earth rotation). It is important to keep this fact in mind, since we will need to refer often to the frequency of the nutation as well that of the associated wobble and polar motion.
The terms low frequency, diurnal, semidiurnal, $\cdots$ will be used herein for frequencies (of wobbles, nutations, or polar motions, as the case may be) within bands of width 1 cpsd centered at $0, \pm 1, \pm 2, \cdots$ cpsd, as seen in the TRF for wobbles and polar motions, and in the CRF for nutations. Positive (negative) frequencies refer to prograde (retrograde) motions in the relevant reference frame.

High frequency nutations result from the action of the tide generating potential on elements of the Earth's density distribution that give rise to geopotential coefficients $\left(C_{k, l}, S_{k, l}\right)$ with $l>0$. Rigid Earth nutations relating to the cases $k=3,4$ have been the special focus of Folgueira et al. (1998a). A listing of diurnal and subdiurnal nutations with coefficients down to a few hundredths of $1 \mu$ as may be found in Folgueira et al. (2001), with comparisons to values obtained by others. Now that realistic uncertainties as low as $5 \mu$ as have become possible in the estimation of many nutation components (see, for instance, Herring et al. 2002), high frequency nutations - at least, those with amplitudes of several $\mu$ as - have to be taken seriously from the observational point of view. Theoretical evaluation of the amplitudes of the high frequency nutations of the nonrigid Earth is therefore of considerable interest. (For recent results on low frequency nutations of the nonrigid Earth, see Mathews et al. 2002 and references cited therein.) Bizouard et al. (2001) have presented numbers for the coefficients of nutations of diurnal and semidiurnal frequencies. Their results for the nonrigid Earth have been obtained by applying (an early version of) the transfer function of Mathews et al. (2002) to the rigid Earth numbers from the works cited above. However, as will be seen from the theoretical development to be presented in later sections, the use of that transfer function, which was constructed for the low frequency nutations, is inappropriate in principle for high frequency nutations. Semidiurnal nutations of the nonrigid Earth have been computed by Getino et al. (2001). Their results are consistent with the transfer function being essentially constant across the semidiurnal band. We shall show from very simple physical considerations that the transfer function should be very nearly equal to the ratio of the moment of inertia of the whole Earth to that of the mantle; the complicated formalism used by the above authors yields no such insights. Escapa et al. (2002) have drawn attention to a contribution to the semidiurnal nutations from the resonance in the retrograde diurnal wobbles related to the free core nutation, via the Earth's triaxiality. The approach used in the present work makes the mechanism responsible for this contribution quite transparent.

We begin with a systematic presentation, in Sect. 2, of the interrelations of wobbles, polar motions, and nutations induced by a spectral component of the tidal gravitational potential. General expressions for the torque exerted on the Earth by the potential of arbitrary spherical harmonic type $(n, m)$, not found in the existing literature, are presented in Sect. 3. (The "type" $(n, m)$ refers to the spherical harmonic degree $n$ and order $m$ of the potential.) These expressions, in which separate prograde and retrograde parts appear, are fundamental to this work, and are derived in Appendix A.

Section 4 deals with the dynamical equations governing the wobble motion for a two-layer Earth composed of the mantle 
and the fluid core which are mutually coupled; the equations are an adaptation of those of Sasao et al. (1980) to forcing by potentials of general type $(n, m)$. They are entirely adequate for the treatment of all except the retrograde diurnal wobbles with which the low frequency (LF) nutations are associated. The LF nutations, which are by far the most dominant, call for very detailed modeling and are specifically excluded from the ambit of this paper; see Mathews et al. (2002) for a comprehensive treatment which includes the inner core and various other effects in the modeling. Certain enhancements made in that work, like inclusion of mantle anelasticity and ocean tide effects, are retained here as they are of relevance in the case of the prograde diurnal nutations. The core-mantle electromagnetic coupling, also considered in that paper, is insignificant in the present context.

The general solution of the dynamical equations is given in Sect. 5. The LF polar motions and the prograde diurnal ones require special consideration; they are dealt with in Sects. 6 and 7 , respectively. The special features in the former case are the existence of the Chandler resonance in the LF band, and the influence of mantle anelasticity on the frequency of the resonance. In the latter case, a coupling between prograde and retrograde wobbles, arising from the triaxiality terms in the angular momentum, has to be taken into account; it is of no consequence in the any other case. It is instructive (and convenient) to take advantage of this coupling for computation of the amplitudes of prograde diurnal polar motions by establishing and making use of their relation to the known amplitudes of the low frequency nutations excited by the same tidal potential; we shall do so in Sect. 7. The possible contribution from triaxiality of the fluid core is of special interest, and is investigated.

Section 8 begins with the explicit expressions for the coefficients of circular polar motions, and their interrelations. General relations connecting coefficients of circular nutations to those of the equivalent polar motions are deduced. Numerical values from computations based on our theoretical approach are presented in several tables of polar motions and/or equivalent nutations in a number of prograde and retrograde frequency bands. Comparisons of our values are made with results from earlier works, for circular motions or for elliptical ones, as available. Finally our numerical results for the contributions from possible triaxiality of the Earth's core are compared with those of Escapa et al. (2002). The concluding section summarizes the main results, highlighting some special features. In particular, we discuss the possibility of determining the trixiality of the core from observations of prograde diurnal polar motions and conclude that the prospects are dim.

\section{Wobble, nutation, and polar motion: Kinematics}

\subsection{Wobbles}

The wobbles of the mantle and the fluid core are described by $\boldsymbol{m}(t)$ and $\boldsymbol{m}_{\mathrm{f}}(t)$, defined in terms of the instantaneous angular velocity vectors $\boldsymbol{\Omega}, \boldsymbol{\Omega}_{\mathrm{f}}$ of the respective regions by

$\boldsymbol{\Omega}=\Omega_{0}\left[\left(1+m_{3}\right) \boldsymbol{i}_{3}+\boldsymbol{m}(t)\right], \boldsymbol{\Omega}_{\mathrm{f}}-\mathbf{\Omega}=\Omega_{0}\left[m_{f 3} \boldsymbol{i}_{3}+\boldsymbol{m}_{\mathrm{f}}(t)\right]$, where $\boldsymbol{i}_{3}$ is the axis of maximum moment of inertia of the Earth, $\Omega_{0}$ is mean angular velocity of Earth rotation, equivalent to $1 \mathrm{cpsd}$, and $m_{3}$ and $\left(m_{3}+m_{f 3}\right)$ represent the fractional variations in the axial spin rates of the mantle and the core, respectively. As is well known, use of the complex combinations

$\tilde{m}(t) \equiv m_{1}(t)+\mathrm{i} m_{2}(t), \quad$ and $\quad \tilde{m}_{\mathrm{f}}(t) \equiv m_{f 1}(t)+\mathrm{i} m_{f 2}(t)$

of the components of $\boldsymbol{m}(t)$ and $\boldsymbol{m}_{\mathrm{f}}(t)$ helps to express the dynamical equations of the wobbles compactly. Their individual spectral components have the forms

$\tilde{m}(t)=\tilde{m}(\sigma) \mathrm{e}^{\mathrm{i}\left(\sigma \Omega_{0} t+\alpha_{\sigma}\right)}, \quad \tilde{m}_{\mathrm{f}}(t)=\tilde{m}_{\mathrm{f}}(\sigma) \mathrm{e}^{\mathrm{i}\left(\sigma \Omega_{0} t+\alpha_{\sigma}\right)}$,

where $\sigma$ is the frequency (in cpsd) and $\alpha_{\sigma}$ is the phase, of the wobble; $\sigma$ is positive (negative) for prograde (retrograde) wobbles.

The phase of a wobble due to lunisolar forcing is related, of course, to the argument of the relevant spectral component of the lunisolar potential. In the convention of Cartwright \& Tayler (1971), the potential of spherical harmonic type $(n, m)$ and frequency $\omega$ at the point with colatitude $\theta$ and longitude $\lambda$ at a geocentric distance $r$ is expressed as

$V_{\omega}^{(n, m)}(\boldsymbol{r} ; t)=g_{\mathrm{e}} H_{\omega}^{(n, m)}\left(r / a_{\mathrm{e}}\right)^{n} \operatorname{Re}\left[Y_{n}^{m}(\theta, \lambda) \mathrm{e}^{\mathrm{i}\left(\Theta_{\omega}(t)-\zeta_{n, m}\right)}\right]$,

where $a_{\mathrm{e}}$ is the Earth's equatorial radius, $g_{\mathrm{e}}=G M_{\mathrm{E}} / a_{\mathrm{e}}^{2}\left(M_{\mathrm{E}}\right.$ being the Earth's mass), and $H_{\omega}^{(n, m)}$ is the amplitude (expressed as a height) and $\Theta_{\omega}(t)$ is the argument of this spectral component of the tidal potential. The role of $\zeta_{n, m}$, defined by

$\zeta_{n, m}=0$ for $(n-m)$ even and

$\zeta_{n, m}=\pi / 2$ for $(n-m)$ odd,

is to ensure that the potential involves $\cos \Theta_{\omega}(t)$ or $\sin \Theta_{\omega}(t)$ according as $(n-m)$ is even or odd, in conformity with the Cartwright-Tayler convention. The argument $\Theta_{\omega}(t)$ is expressed as a linear combination, with integer coeffients, of the Doodson's fundamental tidal arguments $\left(\tau-\lambda, s, h, p, N^{\prime}, p_{s}\right)$, with $m$ as the coefficient of the first of these. It is useful to note that $\tau+s-\lambda=G M S T+\pi$, GMST being the Greenwich Mean Sidereal Time (in radians). For all practical purposes, $\Theta_{\omega}(t)$ is a linear function of time, and

$\omega=\frac{\mathrm{d} \Theta_{\omega}(t)}{\mathrm{d} t}$

with $\omega$ confined, for given $m$, to the interval

$0 \leq \omega<(1 / 2) \Omega_{0}, \quad(m=0) ; \quad$ and

$(m-1 / 2) \Omega_{0}<\omega<(m+1 / 2) \Omega_{0}, \quad(m>0)$.

The torque exerted by the potential (4) on the Earth, which is considered in detail in the next section, has both prograde and retrograde components. The relation between the argument $\Theta_{\omega}(t)$ and the phases $\left(\sigma \Omega_{0} t+\alpha_{\sigma}\right)$ of the wobbles produced by the respective components of the torque is

$\sigma \Omega_{0} t+\alpha_{\sigma}= \pm\left(\Theta_{\omega}(t)-\zeta_{n, m}\right), \quad \sigma= \pm\left(\omega / \Omega_{0}\right)$,

wherein the upper (lower) signs are for the prograde (retrograde) wobbles. 
As we shall show in Sect. 6, an unusual situation arises in the case of the nonrigid Earth if the driving torque is time independent $(\sigma=0)$ : the solution is then not a special case of (3), but is linear in $t$ :

$\tilde{m}(t)=\mathrm{i} \Omega_{0} K t+K^{\prime}$.

\subsection{Nutations}

The complex nutation variable $\tilde{\eta}(t)$, defined by

$\tilde{\eta}(t) \equiv \Delta \psi(t) \sin \epsilon+\mathrm{i} \Delta \epsilon(t)$,

is kinematically related to the mantle wobble variable $\tilde{m}(t)$ :

$\mathrm{i} \frac{\mathrm{d} \tilde{\eta}(t)}{\mathrm{d} t}=\Omega_{0} \tilde{m}(t) \mathrm{e}^{\mathrm{i} \Omega_{0}\left(t-t_{0}\right)}$,

where $-t_{0}$ (or $-\Omega_{0} t_{0}$ in angle units) is $G M S T_{0}$, the Greenwich Mean Sidereal Time (GMST) at the epoch chosen as the origin of time $(t=0)$. It is conventional, in both tidal and nutation theories, to take this epoch to be J2000, i.e., 12 hrs UT1 on January 1, 2000, and we follow this convention.

$\Omega_{0}\left(t-t_{0}\right)=\Omega_{0} t+G M S T_{0}=G M S T$,

where $G M S T_{0}=4.894961212$ radians.

Equation (11) is a linear approximation to the exact relation, and is entirely adequate for the present purposes. Taken together with Eqs. (3) and (12), it implies that a wobble of frequency $\sigma$ cpsd ( $\sigma \neq-1$ or 0 ) has the associated nutation of frequency $v$ cpsd:

$$
\begin{aligned}
\tilde{\eta}(t) & =-\frac{\tilde{m}(\sigma)}{1+\sigma} \mathrm{e}^{\mathrm{i}\left[\nu \Omega_{0} t+\alpha_{\sigma}+G M S T_{0}\right]} \\
& =-\frac{\tilde{m}(\sigma)}{1+\sigma} \mathrm{e}^{\mathrm{i \textrm {i } ( \Theta _ { \omega } ( t ) - \zeta _ { n , m } ) + \mathrm { i } G M S T},}
\end{aligned}
$$

where, in view of the second of Eqs. (8),

$v=1+\sigma=1 \pm \omega / \Omega_{0}$.

The plus (minus) sign in the above equation is for prograde (retrograde) wobbles.

Now, the conventional argument $\Xi_{\gamma}$ of a nutation of frequency $v$ is related to the argument $\Theta_{\omega}(t)$ of the tide which excites the nutation through

$\Xi_{v}= \pm\left(\Theta_{\omega}(t)-m \pi\right)+G M S T$

$\mathrm{e}^{\mathrm{i} \Xi_{\nu}}=(-1)^{m} \mathrm{e}^{ \pm \mathrm{i} \Theta_{\omega}(t)+\mathrm{i} G M S T}$.

Therefore the expression (13) for $\tilde{\eta}(t)$ becomes, for a nutation excited by a tidal potential of order $m$,

$$
\begin{aligned}
& \tilde{\eta}(t)=(-1)^{m} \tilde{\eta}(v) \mathrm{e}^{\mathrm{i} \Xi_{v}(t) \mathrm{F} \xi_{n, m},} \\
& \tilde{\eta}(v)=\frac{-\tilde{m}(\sigma)}{1+\sigma} .
\end{aligned}
$$

The relations (15) give rise to the factor $(-1)^{m}$ which appears in (16). This factor which would have been missed if the constants in the phases of the wobbles and nutations were not explicitly kept track of, and the factor $\mathrm{e}^{\mathrm{F} \mathrm{i}_{n, m}}$ (which is 1 or $\mp \mathrm{i}$ according as $n-m$ is even or odd), play essential roles in correctly identifying the coefficients of $\cos \Xi_{v}(t)$ and $\sin \Xi_{v}(t)$ computed from the tidal potential, and in ensuring that they have the correct signs.

The special case $\sigma=-1$ leads to a secularly varying $\tilde{\eta}(t)$, representing precession:

$\tilde{\eta}(t)=\tilde{m}(-1) \mathrm{i} \Omega_{0} t+\tilde{\eta}(0)$.

\subsection{Polar motion}

Polar motion is represented by

$\tilde{p}(t) \equiv x_{\mathrm{p}}(t)-\mathrm{i} y_{\mathrm{p}}(t)=-\tilde{\eta}(t) \mathrm{e}^{-\mathrm{i} G M S T}$,

as has been made explicit by Gross (1992) and Brzeziński \& Capitaine (1993). It is evident that its frequency is $(v-1)=$ $\sigma$ cpsd, i.e., the same as that of the associated wobble. One obtains, on using (13),

$$
\begin{aligned}
& \tilde{p}(t)=\tilde{p}(\sigma) \mathrm{e}^{\mathrm{i}\left(\sigma \Omega_{0} t+\alpha_{\sigma}\right)}=\tilde{p}(\sigma) \mathrm{e}^{ \pm \mathrm{i}\left(\Theta_{\omega}(t)-\zeta_{n, m}\right)}, \\
& \tilde{p}(\sigma)=\frac{\tilde{m}(\sigma)}{(1+\sigma)} .
\end{aligned}
$$

In the special case when the torque is time independent $(\sigma=0)$, one finds on integrating Eq. (11) with $\tilde{m}(t)$ taken from (9), and then using (19), that

$\tilde{p}(t)=\mathrm{i} K \Omega_{0} t+\left(K^{\prime}-K\right)+K^{\prime \prime} \mathrm{e}^{-\mathrm{i} \Omega_{0} t}$,

where $K^{\prime}$ and $K^{\prime \prime}$ involve the intial values of $\tilde{m}$ and $\tilde{p}$. The specifics of this case are dealt with in Sect. 6. The nutation corresponding to (22a) is

$\tilde{\eta}(t)=-\mathrm{i} K \Omega_{0} t \mathrm{e}^{\mathrm{i} G M S T}$,

with the omission of the initial value terms. A nutation of this type, which is periodic but with an amplitude varying linearly with time, appears to have been not encountered before.

\section{Dynamics: The torque}

Evaluation of the torque $\boldsymbol{\Gamma}_{\omega}^{(n, m)}$ on the Earth due to the potential $V_{\omega}^{(n, m)}$ of Eq. (4) is done in Appendix A for general $(n, m)$. Though the potential wave is strictly retrograde, the complex combination

$\tilde{\Gamma}_{\omega}^{(n, m)} \equiv\left(\Gamma_{\omega, 1}^{(n, m)}+\mathrm{i} \Gamma_{\omega, 2}^{(n, m)}\right)$

of the equatorial components of the torque $\boldsymbol{\Gamma}_{\omega}^{(n, m)}$ is seen to be composed of both prograde and retrograde parts:

$\tilde{\Gamma}_{\omega}^{(n, m)}(t)=\tilde{\Gamma}_{\omega}^{(n, m)}\left(\sigma_{\mathrm{p}}\right) \mathrm{e}^{\mathrm{i}\left(\Theta_{\omega}(t)-\zeta_{n, m}\right)}+\tilde{\Gamma}_{\omega}^{(n, m)}\left(\sigma_{\mathrm{r}}\right) \mathrm{e}^{-\mathrm{i}\left(\Theta_{\omega}(t)-\zeta_{n, m}\right)}$,

where the subscripts $\mathrm{p}$ and $\mathrm{r}$ denote "prograde" and "retrograde", respectively. The dependence of $\Theta_{\omega}(t)$ and $-\Theta_{\omega}(t)$ on time is given by Eq. (8), and

$\sigma_{\mathrm{p}}=\omega / \Omega_{0}, \quad \sigma_{\mathrm{r}}=-\omega / \Omega_{0}=-\sigma_{\mathrm{p}}$.

It follows now from Eq. (7) that for the torques due to nonzonal $(m>0)$ potentials, and for the wobbles excited by them, $\sigma_{\mathrm{p}}$ and $\sigma_{\mathrm{r}}$ lie in separate bands of width 1 cpsd each, centered 
at $m$ cpsd and $-m$ cpsd, respectively, while for $m=0$, the prograde and retrograde frequencies together constitute a single band of width $1 \mathrm{cpsd}$ centered at 0 cpsd. The frequency bands of the nutations produced by $V_{\omega}^{(n, m)}$ are shifted by +1 cpsd relative to those of the associated wobbles, in view of (14).

We present now explicit expressions for the amplitudes $\tilde{\Gamma}_{\omega}^{(n, m)}\left(\sigma_{\mathrm{p}}\right)$ and $\tilde{\Gamma}_{\omega}^{(n, m)}\left(\sigma_{\mathrm{r}}\right)$ (see Appendix A). The retrograde amplitude has distinct forms for the zonal $(m=0)$ and nonzonal cases.

$$
\begin{aligned}
\tilde{\Gamma}_{\omega}^{(n, m)}\left(\sigma_{\mathrm{p}}\right)= & \left(\mathrm{i} \Omega_{0}^{2} \bar{A}\right)(-1)^{m+1} G_{n, m}^{(+)} H_{\omega}^{(n, m)} \\
& \times\left(C_{n, m+1}+\mathrm{i} S_{n, m+1}\right),(0 \leq m<n), \\
\tilde{\Gamma}_{\omega}^{(n, m)}\left(\sigma_{\mathrm{r}}\right)= & \left(\mathrm{i} \Omega_{0}^{2} \bar{A}\right)(-1)^{m} G_{n, m}^{(-)} H_{\omega}^{(n, m)} \\
& \times\left(C_{n, m-1}-\mathrm{i} S_{n, m-1}\right),(1 \leq m \leq n), \\
\tilde{\Gamma}_{\omega}^{(n, 0)}\left(\sigma_{\mathrm{r}}\right)= & -\left(\mathrm{i} \Omega_{0}^{2} \bar{A}\right) G_{n, 0}^{(+)} H_{\omega}^{(n, 0)}\left(C_{n, 1}+\mathrm{i} S_{n, 1}\right) \\
= & \tilde{\Gamma}_{\omega}^{(n, 0)}\left(\sigma_{\mathrm{p}}\right),
\end{aligned}
$$

wherein $\bar{A} \equiv(A+B) / 2$ stands for the mean equatorial moment of inertia, and $G_{n, m}^{(+)}$and $G_{n, m}^{(-)}$are given by

$$
\begin{aligned}
G_{n, m}^{(+)} & =(n-m)(n+m+1) G_{n, m}, \quad G_{n, m}^{(-)}=\frac{2}{\left(2-\delta_{m, 1}\right)} G_{n, m}, \\
G_{n, m} & =\left(\frac{2 n+1}{4 \pi} \frac{(n+m) !}{(n-m) !}\right)^{1 / 2} \frac{g_{\mathrm{e}} M_{\mathrm{E}}}{4 \Omega_{0}^{2} \bar{A}} .
\end{aligned}
$$

The expressions (26) show that the prograde part of the torque is due to the action of $V_{\omega}^{(n, m)}$ on $C_{n, m+1}$ and $S_{n, m+1}$, and the retrograde part is, for all $m>0$, due to its action on $C_{n, m-1}$ and $S_{n, m-1}$. In the case of zonal potentials, both the prograde and the retrograde torques result from the action on $C_{n, 1}$ and $S_{n, 1}$.

In the special case of the constant term $(\omega=0)$ present in the spectrum of any zonal potential of even order $n, \Theta_{\omega}(t)=0$, and the expression (24) for the torque reduces to

$\tilde{\Gamma}_{0}^{(n, 0)}(t)=-2\left(\mathrm{i} \Omega_{0}^{2} \bar{A}\right) G_{n, 0}^{(+)} H_{0}^{(n, 0)}\left(C_{n 1}+\mathrm{i} S_{n 1}\right)$,

( $n$ even) (28)

on using the relevant expressions from Eqs. (26). The nutation due to this term requires special consideration, as will be seen in Sect. 6.

It should be noted that torques due to the degree 2 sectorial $(m=2)$ and zonal potentials are ignorable because $C_{2,1}$ and $S_{2,1}$ are.

The origin of nutations and polar motions in the various frequency bands, as displayed in Table 1, is clear from the above considerations.

To compute the nutations or polar motions in a particular band of frequencies, one starts by identifying from Table 1 the types of potentials and geopotential coefficients relevant to that band, then picking out the expressions for the torques which they produce, and finally, solving the dynamical equations with these as the driving torques. For prograde semidiurnal nutations (or prograde diurnal polar motions), for instance, the relevant potentials are of type $(n, 1)$, acting on $C_{n, 2}$ and $S_{n, 2}$. The values of the geopotential coefficients of relevance to this work are shown in Table 2. The first set (labeled IERS92) gives the values from McCarthy (1992), used by Bretagnon et al. (1997) and other workers in computing the high frequency nutations of the rigid Earth, and the second set lists the JGM3 (Joint Gravity Model 3) values transformed to the same normalization as IERS92. We use the latter (more recent) set for our computations for the nonrigid Earth.

\section{The dynamical equations}

Since the amplitudes of the HF nutations are expected to be no more than about $20 \mu$ as, the effects of the solid inner core are expected to be negligible at the level of $0.1 \mu$ as, which is the accuracy we aim for. So we treat the Earth as consisting of only two regions - the mantle and a fluid core.

The coupled rotational motions of of these two regions which are excited by potentials of any type $(n, m) \neq(2,1)$ are governed by dynamical equations of the Sasao et al. (1980) form for the wobble variables $\tilde{m}(t)$ and $\tilde{m}_{\mathrm{f}}(t)$. They are, in the time domain,

$$
\left(\frac{\mathrm{d}}{\mathrm{d} t}-\mathrm{i} e \Omega_{0}\right) \tilde{m}(t)+\frac{1}{A}\left(\frac{\mathrm{d}}{\mathrm{d} t}+\mathrm{i} \Omega_{0}\right)\left[\tilde{c}_{3}(t)+A_{\mathrm{f}} \tilde{m}_{\mathrm{f}}(t)\right]=\frac{\tilde{\Gamma}(t)}{A \Omega_{0}},\left(2 \theta_{2}\right.
$$

$\left(\frac{\mathrm{d}}{\mathrm{d} t}+\mathrm{i} \Omega_{0}\left(1+e_{\mathrm{f}}\right)\right) \tilde{m}_{\mathrm{f}}(t)+\frac{\mathrm{d}}{\mathrm{d} t}\left(\tilde{m}(t)+\frac{\tilde{c}_{3}^{\mathrm{f}}(t)}{A_{\mathrm{f}}}\right)=0$.

Here $\tilde{\Gamma}(t)$ stands for $\tilde{\Gamma}_{\omega}^{(n, m)}(t) ; e, e_{\mathrm{f}}$ are the ellipticities of the whole Earth and of the fluid core, and $\tilde{c}_{3}(t), \tilde{c}_{3}^{\mathrm{f}}(t)$ are complex combinations,

$\tilde{c}_{3}=c_{1,3}+\mathrm{i} c_{2,3} \quad$ and $\quad \tilde{c}_{3}^{\mathrm{f}}=c_{1,3}^{\mathrm{f}}+\mathrm{i} c_{2,3}^{\mathrm{f}}$

of the indicated elements of their respective inertia tensors. The mean equatorial moments of inertia $\bar{A}, \bar{A}_{\mathrm{f}}$, and $\bar{A}_{\mathrm{m}}$ of the Earth and of its core and mantle are written here simply as $A, A_{\mathrm{f}}, A_{\mathrm{m}}$; the overbars will be restored in Sect. 7 where triaxiality needs to be taken into account.

The quantity $\tilde{c}_{3}$ in (30) represents the deformation of type $(2,1)$ of the whole Earth due to the direct action of the tidal potential, together with that due to incremental centrifugal potentials associated with the wobbles $\tilde{m}$ and $\tilde{m}_{\mathrm{f}}$. The origins of $\tilde{c}_{3}^{\mathrm{f}}$ are similar, but the relevant deformation is that of the core alone. For a spherically symmetric Earth, the deformation induced by a tidal potential of type $(n, m)$ is strictly of the same type, and will therefore contribute nothing to $\tilde{c}_{3}$ or $\tilde{c}_{3}^{\mathrm{f}}$ unless $(n, m)=(2,1)$. Although the ellipticity of the Earth would result in the presence of a small part (of the order of 1/300) of type $(2,1)$ in the deformation due to $(n, 1)$ potentials with $n \neq 2$, its effect on the already small nutations produced by such potentials is entirely negligible. However, the incremental centrifugal potentials are necessarily of type $(2,1)$ irrespective of the nature of the tidal potential, and so is its contribution to deformation. Consequently, the expressions of Sasao et al. (1980) for $\tilde{c}_{3}$ and $\tilde{c}_{3}^{\mathrm{f}}$ take the following modified form in the general case:

$\tilde{c}_{3}=A\left[\kappa\left(\tilde{m}-\tilde{\phi} \delta_{n, 2} \delta_{m, 1}\right)+\xi \tilde{m}_{\mathrm{f}}\right]$,

$\tilde{c}_{3}^{\mathrm{f}}=A_{\mathrm{f}}\left[\gamma\left(\tilde{m}-\tilde{\phi} \delta_{n, 2} \delta_{m, 1}\right)+\beta \tilde{m}_{\mathrm{f}}\right]$, 
Table 1. Origin of nutations, wobbles, and polar motions in different frequency bands.

\begin{tabular}{lcccl}
\hline \hline Nutations & $\begin{array}{c}\text { Frequency } \\
\text { band (cpsd) }\end{array}$ & $\begin{array}{c}\text { due to } \\
\text { action }\end{array}$ & $\begin{array}{c}\text { by potentials } \\
\text { of type }\end{array}$ & $\begin{array}{l}\text { Wobbles \& } \\
\text { Polar motions }\end{array}$ \\
\hline Long period & $(-0.5,+0.5)$ & $C_{n, 0}$ & $(n, 1)$ & Retro diurnal \\
Pro diurnal & $(+0.5,+1.5)$ & $\left(C_{n, 1}, S_{n, 1}\right)$ & $(n, 0)$ & Long period \\
Retro diurnal & $(-1.5,-0.5)$ & $\left(C_{n, 1}, S_{n, 1}\right)$ & $(n, 2)$ & Retro semidiurnal \\
Pro semidiurnal & $(+1.5,+2.5)$ & $\left(C_{n, 2}, S_{n, 2}\right)$ & $(n, 1)$ & Pro diurnal \\
Retro semidiurnal & $(-2.5,-1.5)$ & $\left(C_{n, 2}, S_{n, 2}\right)$ & $(n, 3)$ & Retro terdiurnal \\
Pro terdiurnal & $(+2.5,+3.5)$ & $\left(C_{n, 3}, S_{n, 3}\right)$ & $(n, 2)$ & Pro semidiurnal \\
\hline
\end{tabular}

where $\tilde{\phi}$ is equivalent to the tidal potential but is expressed in suitable dimensionless units, and $\kappa, \xi, \gamma$, and $\beta$ are compliance (deformability) parameters (see Sasao et al. 1980; Mathews et al. 1991). The Kronecker delta function $\delta_{p, q}$ is unity if $p=q$, and is zero otherwise.

There is a caveat to be entered in regard to the above expressions, because the compliances have to be considered to be frequency dependent, in general, even within a particular tidal band. The reason is that the deformation due to ocean tides raised by the tidal potential, and the effect of mantle anelasticity on the deformations, are both reflected in the values of the compliances - and one or the other of these could be strongly frequency dependent, depending on the type $(n, m)$ of the potential involved (see, for instance, Mathews et al. 2002). This dependence, to the extent that it is known, can be readily taken into account if the expressions (31) are taken in the frequency domain, i.e., as expressions for $\tilde{c}_{3}(\sigma)$ and $\tilde{c}_{3}^{\mathrm{f}}(\sigma)$ in terms of the spectral amplitudes $\tilde{m}(\sigma), \tilde{m}_{\mathrm{f}}(\sigma)$, and $\tilde{\phi}(\sigma)$; this is what we do.

The frequency domain version of Eqs. (29) is now readily obtained by replacing $\mathrm{d} / \mathrm{d} t$ by $\mathrm{i} \Omega_{0} \sigma$ and introducing the expressions (31). One should keep in mind that $\tilde{\Gamma}$ here stands for $\tilde{\Gamma}_{\omega}^{(n, m)}$, which has both prograde and retrograde parts. For any term belonging to the prograde part, $\sigma$ is to be taken as $\sigma_{\mathrm{p}} \equiv \omega / \Omega_{0}$, and $\tilde{\Gamma}(\sigma)$ is given by (26a), while for terms in the retrograde part, $\sigma$ is $\sigma_{\mathrm{r}} \equiv-\omega / \Omega_{0}$ and $\tilde{\Gamma}(\sigma)$ is given by (26b) or (26c) according as $m \neq 0$ or $m=0$. With this understanding, we continue to use the generic symbol $\sigma$ in writing down the pair of frequency domain equations for the wobbles due to a tidal perturbation of general type $(n, m) \neq(2,1)$ :

$$
\begin{aligned}
& {[(\sigma-e)+(1+\sigma) \kappa] \tilde{m}(\sigma)+(1+\sigma)\left(\xi+A_{\mathrm{f}} / A\right) \tilde{m}_{\mathrm{f}}(\sigma)} \\
& =\tilde{\Gamma}(\sigma) /\left(\mathrm{i} A \Omega_{0}^{2}\right), \\
& (1+\gamma) \sigma \tilde{m}(\sigma)+\left[1+\mathrm{e}_{\mathrm{f}}+(1+\beta) \sigma\right] \tilde{m}_{\mathrm{f}}=0 .
\end{aligned}
$$

It is a pertinent to ask, at this point, whether the nonlinear terms in the dynamical equations (Mathews et al. 2002), which had been ignored so far, could contribute to $\tilde{p}(\sigma) \equiv \tilde{m}(\sigma) /(1+\sigma)$ at the $0.1 \mu$ as level. As may be seen from the Appendix A of that paper, the largest of the contributions to $\tilde{m}(t)$ from nonlinear terms are $\left(c_{1,1}-c_{3,3}\right)^{(Z)} \tilde{\phi} / A$ and $\left(c_{1,1}+\mathrm{i} c_{1,2}\right)^{(S)} \tilde{\phi}^{*} / A$, wherein all the quantities are in the time domain; $\tilde{\phi}$ is the tesseral tidal potential, and the inerita tensor elements involved arise from the action of the zonal or sectorial tides, as identified by the superscript $Z$ or $S$. The $\left(c_{i, j} / A\right)$ are of the order of $\kappa \phi^{(Z)}$ and $\kappa \tilde{\phi}^{(S)}$ in the two cases. Since $\kappa \approx 10^{-3}$ and the largest of the spectral components of the various potentials are of $O\left(10^{-5}\right)$, it would appear that contributions to $\tilde{m}(\sigma)$ of the order of $10^{-13}$ radians, i.e., about $0.1 \mu \mathrm{as}$, could arise. The fact that this does happen in the case of the retrograde diurnal wobbles is not relevant, however, for wobbles in the other bands. The reason is that the frequency spectrum of $c_{i, j}^{(Z)}$ lies in the low frequency band and that of $c_{i, j}^{(S)}$ is in the retrograde semidiurnal band, while the spectra of $\tilde{\phi}$ and $\tilde{\phi}^{*}$ are in the retrograde and prograde diurnal bands respectively. It follows then then the spectra of the above-mentioned contributions to $\tilde{m}(t)$ are only in the retrograde diurnal band and are of no consequence for the other bands with which this work is concerned. Therefore the use of the linear Eqs. (29) or (32) for our purposes is entirely justified.

\section{Solution of the dynamical equations}

The formal solution of the pair of linear Eqs. (32) is nearly trivial. Denoting the secular determinant of this system by $D(\sigma)$ and the coefficient $\left[1+e_{\mathrm{f}}+(1+\beta) \sigma\right]$ of $\tilde{m}_{\mathrm{f}}(\sigma)$ in the second equation by $X(\sigma)$, we have

$$
\begin{aligned}
& \tilde{m}(\sigma)=\frac{X(\sigma)}{D(\sigma)} \frac{\tilde{\Gamma}(\sigma)}{\mathrm{i} \Omega_{0}^{2} A}, \\
& D(\sigma)=\left(A_{\mathrm{m}} / A\right)\left(\sigma-\sigma_{1}\right)\left(\sigma-\sigma_{2}\right),
\end{aligned}
$$

where $A_{\mathrm{m}}$ is the mean equatorial moment of inertia of the mantle, and $\sigma_{1}$ and $\sigma_{2}$ are the resonance frequencies associated, respectively, with the Chandler wobble $(\mathrm{CW})$ and the nearly diurnal free wobble corresponding to the FCN. They are given by

$\sigma_{1}=\frac{A}{A_{\mathrm{m}}}(e-\kappa) \quad$ and $\quad \sigma_{2}=-1-\frac{A}{A_{\mathrm{m}}}\left(e_{\mathrm{f}}-\beta\right)$

to the first order in the ellipticity and compliance parameters, none of which exceeds 1/300 in magnitude. Quantities of this order of magnitude will be referred to as of $O(\epsilon)$. The retrograde diurnal wobble frequency band centered at $\sigma=-1$ corresponds to the low frequency nutations which we have expressly excluded from consideration here. The frequencies of the wobbles corresponding to high frequency nutations are all far from $-1 \mathrm{cpsd}$. Therefore the small parameters referred to above may be neglected in comparison with $(\sigma+1)$; they may also be dropped from $X$ for similar reasons. Then $\left(\sigma-\sigma_{2}\right) \approx$ $(\sigma+1)$, and $X \approx(1+\sigma)$. Hence, the approximation

$\tilde{m}(\sigma)=\frac{A}{A_{\mathrm{m}}} \frac{1}{\left(\sigma-\sigma_{1}\right)} \frac{\tilde{\Gamma}(\sigma)}{\mathrm{i} \Omega_{0}^{2} A}$ 
Table 2. Values of geopotential coefficients.

\begin{tabular}{lcrcr}
\hline \hline & \multicolumn{2}{c}{ IERS92 } & \multicolumn{2}{c}{ JGM3 } \\
$(k, l)$ & \multicolumn{1}{c}{$C_{k, l}$} & $S_{k, l}$ & $C_{k, l}$ & $S_{k, l}$ \\
\hline$(2,2)$ & 1.574410 & -0.903757 & 1.574536 & -0.903868 \\
$(3,1)$ & 2.190181 & 0.269185 & 2.192799 & 0.268012 \\
$(3,2)$ & 0.308936 & -0.211582 & 0.309016 & -0.211402 \\
$(3,3)$ & 0.100447 & 0.197157 & 0.100559 & 0.197201 \\
$(4,1)$ & -0.508638 & -0.449141 & -0.508725 & -0.449460 \\
$(4,2)$ & $\ldots$ & $\ldots$ & 0.350670 & 0.662571 \\
\hline
\end{tabular}

is good for all wobbles outside the retrograde diurnal band. The amplitude of the associated polar motions is then, in view of (21),

$\tilde{p}(\sigma)=\frac{A}{A_{\mathrm{m}}} \frac{1}{(1+\sigma)\left(\sigma-\sigma_{1}\right)} \frac{\tilde{\Gamma}(\sigma)}{\mathrm{i} \Omega_{0}^{2} A}, \quad(|1+\sigma|>1 / 2)$.

If the Earth were rigid, $A / A_{\mathrm{m}}$ would reduce to unity, and $\sigma_{1}$ would be just $e$. With these substitutions, the expression (36) reduces to the wobble amplitude $\tilde{m}_{\mathrm{R}}(\sigma)$ of the rigid Earth. So the transfer function from the rigid to the nonrigid Earth becomes

$T(\sigma) \equiv \tilde{m}(\sigma) / \tilde{m}_{\mathrm{R}}(\sigma)=\frac{A(\sigma-e)}{A_{\mathrm{m}}\left(\sigma-\sigma_{1}\right)}$.

When the excitation frequencies $\sigma$ are outside the low frequency band too, i.e., when $|\sigma|>1 / 2$ (besides $|1+\sigma|>1 / 2$ ), further simplification of (36) and (38) is possible since $e$ and $\sigma_{1}$ are then negligible relative to $|\sigma|$ which dominates by factors of the order of 300 . Thus the transfer function becomes very nearly constant, equal to $\left(A / A_{\mathrm{m}}\right)$; this is the case for all nutations except the low frequency and prograde diurnal ones.

Long period wobbles and polar motions (or the associated prograde diurnal nutations) have to be handled with care, because of the resonance associated with the Chandler wobble whose frequency appears in the low frequency band. These are dealt with in Sect. 6. The zero frequency term in the torque, which pertains to this band, merits special consideration, for reasons to be outlined there.

For numerical computations, which are done to an accuracy of $0.1 \mu$ as and without making use of any of the approximations made above or later, we take $A / A_{\mathrm{m}}=1.1284, e=0.00328455$, and, for an elastic Earth, $\kappa=0.0010505$; the last two are the estimates due to Mathews et al. (2002). The other parameters involved are $e_{\mathrm{f}}=0.0026490, \xi=0.0002248, \gamma=0.0019825$, $\beta=0.0006227$, but they contribute only marginally, if at all, to our numerical results.

\section{Low frequency polar motions and prograde diurnal nutations}

We consider now the motions excited by torques of low frequencies $(|\sigma|<1 / 2)$. The potentials responsible for these torques are of type $(n, 0)$. The nutations produced are below our cut-off level of $0.05 \mu$ as in amplitude for all $n>4$.

\subsection{The Chandler resonance}

The Chandler resonance plays a major role in the wobble response to forcing in this band; so the value of $\kappa$ which appears in the Chandler frequency assumes significance. The effects of mantle anelasticity and ocean tides on nutations can be dealt with by taking into account the complex increments that they produce to the values of $\kappa$ and other compliances, a fact that was exploited by Mathews et al. (2002) in their treatment of low frequency nutations and retrograde diurnal wobbles. In that context, the anelasticity contribution was practically independent of frequency while the ocean tide admittances were strongly frequency dependent, not only due to the FCN resonance, but also because of other aspects of ocean dynamics. In the low frequency tidal band that we are concerned with now, the ocean tides are believed to be essentially equibrium tides, with a constant admittance; but the anelasticity effect varies strongly with frequency across the band, making the anelasticity contribution to $\kappa$ strongly dependent on the forcing frequency. So the apparent frequency of the Chandler resonance, $\sigma_{1}=\left(A / A_{\mathrm{m}}\right)(e-\kappa) \mathrm{cpsd}$, is itself a function of the excitation frequency $\sigma$. More precisely, the polar motion response (37) to forcing at $\sigma$ cpsd is as if there is a resonance at $\sigma=\sigma_{1}(\sigma)$. The Chandler eigenfrequency $\sigma_{\mathrm{CW}}$ (i.e. the frequency of the free Chandler wobble mode) is not variable, of course. It is given by the value of $\sigma_{1}(\sigma)$ for the specific excitation frequency $\sigma$ at which $\operatorname{Re} \sigma_{1}(\sigma)=\sigma$. Another aspect that cannot be ignored is that the imaginary part of the anelasticity contribution to $\kappa$ has to be taken with a sign opposite to that of the forcing frequency: positive for retrograde wobbles and negative for prograde ones. This is required for ensuring that the tidal deformation lags behind the tidal forcing; see Mathews et al. (2002), Appendix C, for details.

\subsection{Anelasticity model and the resonance frequency}

The anelasticity model adopted in this work is the one employed by Mathews et al. (2002). It belongs to the class of models that Wahr \& Bergen (1986) refer to as the $Q_{\mu}$ model of Sailor \& Dziewonski (1978). The essential feature of these models is that the value of a deformational response parameter (e.g., $\kappa)$ of the anelastic Earth to harmonic excitation at some frequency $\omega_{\mathrm{e}}$ differs from the elastic-Earth value at a reference frequency $\omega_{\mathrm{m}}$ by an amount proportional to

$F\left(\omega_{\mathrm{e}} ; \omega_{\mathrm{m}}, \alpha\right)=\cot \left(\frac{\alpha \pi}{2}\right)\left\{1-\left(\frac{\omega_{\mathrm{m}}}{\left|\omega_{\mathrm{e}}\right|}\right)^{\alpha}\right\}-\mathrm{i} s_{\mathrm{e}}\left(\frac{\omega_{\mathrm{m}}}{\left|\omega_{\mathrm{e}}\right|}\right)^{\alpha}$, 
where $s_{\mathrm{e}}=\omega_{\mathrm{e}} /\left|\omega_{\mathrm{e}}\right|$. The reference frequency in the seismic frequency range was taken to be $\omega_{\mathrm{m}}=\left(2 \pi / T_{\mathrm{m}}\right)$ with $T_{\mathrm{m}}=200 \mathrm{~s}$. Compliances of the anelastic Earth in the retrograde diurnal frequency range, computed with $\alpha=0.15$, were found to give optimal results for the low frequency nutations. The same model played a crucial role in the work of Mathews et al. (2002) (see their Appendix D) in demonstrating that their estimate from nutation data for the period of the Chandler resonance (about 383 days) in the Earth's response to forcing at retrograde diurnal frequencies was entirely compatible with the observed Chandler eigenperiod of about 430 days: starting from the former, and taking account of the difference between the ocean tide contributions at the diurnal period of about 1 day and at the observed Chandler period, together with the difference in the anelasticity contributions $\Delta \kappa_{\mathrm{D}}$ and $\Delta \kappa_{\mathrm{CW}}$ to $\kappa$ at these periods as computed from (39), they arrived at the value $\sigma_{\mathrm{CW}}=(2.3175+0.0131 \mathrm{i}) \times 10^{-3}$ for the Chandler eigenferquency. The corresponding period and $Q$ are 430.3 days and 88 respectively. These are quite compatible with estimates from observations of long period polar motions. We felt safe, therefore, in employing this model to compute the anelasticity contribution $\Delta \kappa^{\mathrm{AE}}$ to $\kappa$ as a function of frequency down to the lowest of the wobble frequencies $(\approx 1 / 7000 \mathrm{cpsd})$ relevant to the diurnal nutations listed in the various rigid Earth series cited in the Introduction. If we denote $\left(\omega_{\mathrm{m}} / \omega_{\mathrm{e}}\right)^{\alpha}$ by $f(\sigma)$ for $\omega_{\mathrm{e}}=\Omega_{0} \sigma$ and $f\left(\sigma_{\mathrm{CW}}\right)$ for $\omega_{\mathrm{e}}=\Omega_{0}\left(\operatorname{Re} \sigma_{\mathrm{CW}}\right)$, one can see from (39) that $\Delta \kappa^{\mathrm{AE}}(\sigma)$, the part of $\Delta \kappa(\sigma)$ that is due to anelasticity, may be readily obtained from $\Delta \kappa^{\mathrm{AE}}\left(\sigma_{\mathrm{CW}}\right)$ for which the value $(4.381-1.205 \mathrm{i}) \times 10^{-5}$ has been found in the above-cited work: $\operatorname{Re} \Delta \kappa^{\mathrm{AE}}(\sigma)=\left[(1-f(\sigma)) /\left(1-f\left(\sigma_{\mathrm{CW}}\right)\right)\right] \operatorname{Re} \Delta \kappa^{\mathrm{AE}}\left(\sigma_{\mathrm{CW}}\right)$, and $\operatorname{Im} \Delta \kappa^{\mathrm{AE}}(\sigma)=\left[s_{\sigma} f(\sigma) / f\left(\sigma_{\mathrm{CW}}\right)\right] \operatorname{Im} \Delta \kappa^{\mathrm{AE}}\left(\sigma_{\mathrm{CW}}\right)$, where $s_{\sigma}$ is the sign of $\sigma$. The effective Chandler resonance frequency in the response to excitation at any frequency $\sigma$ in the low frequency band may then be computed as $\sigma_{1}(\sigma)=$ $\sigma_{\mathrm{CW}}+\left(A / A_{\mathrm{m}}\right)\left[\Delta \kappa^{\mathrm{AE}}\left(\sigma_{\mathrm{CW}}\right)-\Delta \kappa^{\mathrm{AE}}(\sigma)\right]$, in view of the expression for $\sigma_{1}$ in (35).

\subsection{Excitation by time independent potential}

It is evident, however, that the model (39) cannot remain valid down to zero frequency: $F$ would become infinite at $\omega=0$, leading to an infinite anelasticity contribution. The zero frequency term present in the $(4,0)$ tidal potential presents, therefore, an exception that cannot be handled by the above procedure. The deformational responses to an incessantly acting potential should actually be characterized by the so-called secular or fluid Love numbers. Now, the compliance $\kappa$ is known to have a simple relation to the $k$ Love number (see, for example, Sasao et al. 1980):

$\kappa=\left(\Omega_{0}^{2} a^{5} / 3 G A\right) k$.

On using for $k$ its fluid (secular) value

$k_{\mathrm{f}}=\left(3 G A / \Omega_{0}^{2} a^{5}\right) e$

which characterizes the response to a time independent degree 2 tidal potential, it follows that $\kappa=e$ for $\omega=0$.

This finding has interesting consequences. One sees trivially that with $\sigma=0$ and $(e-\kappa)=0$, the frequency domain
Eqs. (32) lead to the unphysical result that $\tilde{m}(0)$ is infinite. One has to go back therefore to the time domain Eqs. (29), with $\tilde{c}_{3}(t)$ and $\tilde{c}_{3}^{\mathrm{f}}(t)$ replaced by the expressions (31), noting that the $\tilde{\phi}$ terms drop out since $m=0$ the present case. One sees immediately that the terms proportional to $\tilde{m}(t)$ in Eq. (29a) cancel out as a consequence of the vanishing of $k-e$, and that in the entirely adequate approximation wherein terms of $O(\epsilon)$ are neglected, the two equations then take the forms $\mathrm{d} \tilde{m} / \mathrm{d} t+\left(A_{\mathrm{f}} / A\right) Y=\tilde{\Gamma} /\left(A \Omega_{0}\right)$ and $\mathrm{d} \tilde{m} / \mathrm{d} t+Y=0$, with $Y=\mathrm{d} \tilde{m}_{\mathrm{f}} / \mathrm{d} t+\mathrm{i} \Omega_{0} \tilde{m}_{\mathrm{f}}$. Subtraction of one from the other yields $Y=-\tilde{\Gamma} /\left(A_{\mathrm{m}} \Omega_{0}\right)$, which is a constant in the present case since $\tilde{\Gamma}$ is. The solution for $\tilde{m}(t)$ is then immediate:

$\tilde{m}(t)=\mathrm{i} \Omega_{0} K t+\tilde{m}_{0}, \quad K=\frac{\tilde{\Gamma}(0)}{\mathrm{i} \Omega_{0}^{2} A_{\mathrm{m}}}$

The initial value $\tilde{m}_{0}$ of $\tilde{m}(t)$ is arbitrary. The value of $K$ in the case of the $(4,0)$ potential may be obtained from Eqs. (28) and (27):

$K=-\frac{15}{(4 \pi)^{1 / 2}} \frac{g_{\mathrm{e}} M_{\mathrm{E}}}{\Omega_{0}^{2} A_{\mathrm{m}}} H_{0}^{(4,0)}\left(C_{4,1}+\mathrm{i} S_{4,1}\right)$.

The solution (41) describes a secular motion, relative to the pole of maximum moment of inertia, of the Earth's instantaneous rotation pole. The associated nutation $\tilde{\eta}(t)$, obtained by integrating Eq. (11) after introducing (41), is

$\tilde{\eta}(t)=\left(P-\mathrm{i} K \Omega_{0} t\right) \mathrm{e}^{\mathrm{i} \Omega_{0}\left(t-t_{0}\right)}+Q$,

where $P$ and $Q$ depend on the values $\tilde{m}_{0}$ and $\tilde{\eta}_{0}$ of $\tilde{m}$ and $\tilde{\eta}$ at $t=0$ :

$P=K+\tilde{m}_{0} / \mathrm{i} \Omega_{0}, \quad Q=\tilde{\eta}_{0}-P \mathrm{e}^{-\mathrm{i} \Omega_{0} t_{0}}$.

The "polar motion" $\tilde{p}(t)$ can then be easily seen to be

$$
\begin{aligned}
\tilde{p}(t)= & -\tilde{\eta}(t) \mathrm{e}^{-\mathrm{i} \Omega_{0}\left(t-t_{0}\right)}=\mathrm{i} K \Omega_{0} t-K\left(1-\mathrm{e}^{-\mathrm{i} \Omega_{0} t}\right) \\
& +\left[\tilde{p}_{0} \mathrm{e}^{-\mathrm{i} \Omega_{0} t}-\left(\tilde{m}_{0} / \mathrm{i} \Omega_{0}\right)\left(1-\mathrm{e}^{-\mathrm{i} \Omega_{0} t}\right)\right] .
\end{aligned}
$$

The first term in (45), linear in $t$, being of a type not encountered before, is of considerable interest. It represents a steady drift of the celestial pole in relation to the TRF. The next term, also proportional to the forcing, is of such small magnitude as to be entirely negligible. The remaining terms involve the arbitrary initial values $\tilde{m}_{0}$ and $\tilde{p}_{0}$. Retaining just the linear term, we find that

$x_{\mathrm{p}}(t)=-K_{\mathrm{I}} \Omega_{0} t, \quad y_{\mathrm{p}}(t)=-K_{\mathrm{R}} \Omega_{0} t$,

where the subscripts $\mathrm{R}$ and I represent the real and imaginary parts. $K_{\mathrm{R}}$ and $K_{\mathrm{I}}$ are proportional to $C_{41}$ and $S_{41}$, respectively.

\section{Prograde diurnal polar motions and semidiurnal nutations}

\subsection{Triaxiality contribution to angular momentum}

The prograde semidiurnal nutations arise primarily from the direct action of the $(2,1)$ tidal potential on the Earth's triaxial 
structure. Triaxiality, i.e., inequality of the two principal equatorial moments of inertia $A$ and $B$, is characterized by the parameter $e^{\prime}$ :

$e^{\prime} \equiv \frac{(B-A)}{2 \bar{A}}, \quad$ where $\quad \bar{A} \equiv \frac{A+B}{2}$,

$A=\bar{A}\left(1-e^{\prime}\right), \quad B=\bar{A}\left(1+e^{\prime}\right)$.

The geopotential coefficients $C_{2,2}$ and $S_{2,2}$ are a reflection of triaxiality and are related to $e^{\prime}$ through

$\bar{A} e^{\prime} \cos 2 \alpha=2 M_{\mathrm{E}} a_{\mathrm{E}}^{2} C_{2,2}$,

$\bar{A} e^{\prime} \sin 2 \alpha=2 M_{\mathrm{E}} a_{\mathrm{E}}^{2} S_{2,2}$,

where $\alpha$ is the longitude of the direction of the axis of maximum equatorial moment of inertia. The numbers given by Bretagnon et al. (1997) lead to

$e^{\prime}=1.10157 \times 10^{-5}, \quad \alpha=14.9285$ West.

We define, for later convenience, the complex parameter

$Z=e^{\prime} \mathrm{e}^{2 \mathrm{i} \alpha}=\left(2 M_{\mathrm{E}} a_{\mathrm{e}}^{2} / \bar{A}\right)\left(C_{2,2}+\mathrm{i} S_{2,2}\right)$.

Contributions to the prograde semidiurnal nutations arise from the appearance of triaxiality through $A, B, A_{\mathrm{f}}$, and $B_{\mathrm{f}}$ in the equatorial components of the angular momentum vectors $\boldsymbol{H}$ and $\boldsymbol{H}_{\mathrm{f}}$. In a coordinate system with its axes along the principal axes of the inertia tensor, the equatorial components of $\boldsymbol{H}$ are, for the triaxial Earth,

$H_{1}^{\prime}=A m_{1}^{\prime}=\bar{A}\left(1-e^{\prime}\right) m_{1}^{\prime}, \quad H_{2}^{\prime}=B m_{2}^{\prime}=\bar{A}\left(1+e^{\prime}\right) m_{2}^{\prime}$,

$\tilde{H}^{\prime}(t)=H_{1}^{\prime}(t)+\mathrm{i} H_{2}^{\prime}(t)=\bar{A}\left[\tilde{m}^{\prime}(t)-e^{\prime} \tilde{m}^{\prime *}(t)\right]$.

In reality, the equatorial principal axes make an angle $\alpha$ to the axes of the generally used terrestrial reference frame, and so $\tilde{m}(t)^{\prime}=\mathrm{e}^{-\mathrm{i} \alpha} \tilde{m}(t), \tilde{m}^{\prime *}(t)=\mathrm{e}^{\mathrm{i} \alpha} \tilde{m}^{*}(t)$, and $\tilde{H}^{\prime}(t)=\mathrm{e}^{-\mathrm{i} \alpha} \tilde{H}(t)$, as may be readily seen. It follows then that

$\tilde{H}(t)=\bar{A}\left[\tilde{m}(t)-Z \tilde{m}^{*}(t)\right]$.

If the core is triaxial, $\tilde{H}_{\mathrm{f}}(t)$ also contains triaxiality terms:

$$
\begin{aligned}
\tilde{H}_{\mathrm{f}}(t) & =H_{f 1}(t)+\mathrm{i} H_{f 2}(t) \\
& =\bar{A}_{\mathrm{f}}\left[\left(\tilde{m}(t)+\tilde{m}_{\mathrm{f}}(t)\right)-Z_{\mathrm{f}}\left(\tilde{m}^{*}(t)+\tilde{m}_{\mathrm{f}}^{*}(t)\right)\right], \\
Z_{\mathrm{f}} & =e_{\mathrm{f}}^{\prime} \mathrm{e}^{2 \mathrm{i} \alpha} .
\end{aligned}
$$

The meaning of the symbols used here should be obvious. In using the same angle $\alpha$ here as in the case of $\tilde{H}(t)$, we are assuming that the principal axes of the core have the same directions as those of the whole Earth, which seems the most likely scenario on physical grounds.

\subsection{Wobble equations including triaxiality}

The triaxiality terms appearing through the expressions (52) and (53) had not been included in the dynamical equations in the earlier sections, as the triaxiality contributions to the nutations considered there were far below the $0.1 \mu$ as level because of the weakness of the driving potentials of degree $>2$. With the inclusion of these terms here, the dynamical Eqs. (29) get modified to

$\left(\frac{\mathrm{d}}{\mathrm{d} t}-\mathrm{i} e \Omega_{0}\right) \tilde{m}(t)+\frac{1}{\bar{A}}\left(\frac{\mathrm{d}}{\mathrm{d} t}+\mathrm{i} \Omega_{0}\right)$

$\times\left[\tilde{c}_{3}(t)+\bar{A}_{\mathrm{f}} \tilde{m}_{\mathrm{f}}(t)-\bar{A} Z \tilde{m}^{*}(t)-\bar{A}_{\mathrm{f}} Z_{\mathrm{f}} \tilde{m}_{\mathrm{f}}^{*}(t)\right]=\frac{\tilde{\Gamma}(t)}{\bar{A} \Omega_{0}}$,

$\frac{\mathrm{d}}{\mathrm{d} t}\left(\tilde{m}-Z_{\mathrm{f}} \tilde{m}^{*}+\frac{\tilde{c}_{3}^{\mathrm{f}}(t)}{\bar{A}_{\mathrm{f}}}\right)+\left(\frac{\mathrm{d}}{\mathrm{d} t}+\mathrm{i} \Omega_{0}\left(1+e_{\mathrm{f}}\right)\right) \tilde{m}_{\mathrm{f}}-Z_{\mathrm{f}} \frac{\mathrm{d} m_{\mathrm{f}}^{*}}{\mathrm{~d} t}=0$,

where $\tilde{\Gamma}(t)$ is the full torque due to the $(2,1)$ potential, containing both prograde and retrograde diurnal components.

Consider now the frequency domain version of the above equations, corresponding to a spectral component having the time dependence $\mathrm{e}^{\mathrm{i} \sigma \Omega_{0} t}$. Noting that the amplitude of the term in $\tilde{m}^{*}(t)$ which has this time dependence is $\tilde{m}^{*}(-\sigma)$, and similarly for $\tilde{m}_{\mathrm{f}}^{*}(t)$, one sees that the equations are:

$$
\begin{aligned}
& (\sigma-e) \tilde{m}(\sigma)+(1 / \bar{A})(1+\sigma)\left[\tilde{c}_{3}(\sigma)+\bar{A}_{\mathrm{f}} \tilde{m}_{\mathrm{f}}(\sigma)-\bar{A} Z \tilde{m}^{*}(-\sigma)\right. \\
& \left.-\bar{A}_{\mathrm{f}} Z_{\mathrm{f}} \tilde{m}_{\mathrm{f}}^{*}(-\sigma)\right]=\tilde{\Gamma}(\sigma) /\left(\mathrm{i} \bar{A} \Omega_{0}^{2}\right) \\
& \sigma\left[\tilde{m}(\sigma)+\tilde{c}_{3}^{\mathrm{f}}(\sigma) / \bar{A}_{\mathrm{f}}\right]-\sigma Z_{\mathrm{f}} \tilde{m}^{*}(-\sigma) \\
& +\left(\sigma+1+e_{\mathrm{f}}\right) \tilde{m}_{\mathrm{f}}(\sigma)-\sigma Z_{\mathrm{f}} \tilde{m}_{\mathrm{f}}^{*}(-\sigma)=0 .
\end{aligned}
$$

It is evident that these equations couple the amplitude of any prograde wobble $(\sigma>0)$ to that of the corresponding retrograde wobble with frequency $-\sigma$.

\subsection{Free and forced wobbles}

It is to be expected that the triaxiality terms in Eqs. (55) will lead to small increments to the frequencies of the free wobbles. These increments are of the second order in triaxiality, as may be shown very generally from the structure of these equations. For an elementary illustration, consider the rigid Earth, whose free wobbles are governed by the equation $(\sigma-e) \tilde{m}(\sigma)-(1+\sigma) Z \tilde{m}^{*}(-\sigma)=0$, which is the relevant special case of (55a). On taking its complex conjugate and making the replacement $\sigma \rightarrow-\sigma$, one obtains a second equation relating $\tilde{m}(\sigma)$ and $\tilde{m}^{*}(-\sigma)$. This pair of equations yields the secular equation $\sigma^{2}=\left(e^{2}-e^{\prime 2}\right)$, where $e^{\prime}=|Z|$. Thus the change in the Chandler eigenfrequency due to triaxiality is from $e$ to $e\left(1-e^{\prime 2} / 2 e^{2}\right)$, to the lowest order in the triaxiality. Given the magnitudes of $e$ and $e^{\prime}$, the fractional change is seen to be of the order of $10^{-5}$, which is much too small be of interest. For the Earth with a fluid core, a similar procedure starting with both the Eqs. (55) and their complex conjugates with the sign of $\sigma$ reversed, leads to a similar result also for the second eigenfrequency associated with the free core nutation.

Moving on now to forced prograde diurnal wobbles due to the $(2,1)$ potential, it is advantageous, in view of their coupling to the retrograde diurnals, to express the potential in the form 
employed by Mathews et al. (1991) in their treatment of the retrograde diurnal wobbles:

$\phi^{(2,1)}(\boldsymbol{r} ; t)=-\Omega_{0}^{2}\left[\phi_{1}(t) x z+\phi_{2}(t) y z\right]$.

To obtain the torque due to $\phi^{(2,1)}$, it is simplest to start with (56) reexpressed in the principal axis frame before evaluation of the relevant integrals. The components of the torque can then be transformed back to the original frame. The result, written down for $\tilde{\Gamma}=\boldsymbol{\Gamma}_{1}+\mathrm{i} \boldsymbol{\Gamma}_{2}$, is

$\tilde{\Gamma}=-\mathrm{i} \bar{A} \Omega_{0}^{2}\left[e \tilde{\phi}(t)+Z \tilde{\phi}^{*}(t)\right]$.

Higher order terms which are irrelevant in the present context have been dropped.

For a spectral component of the potential (56) with angular frequency $\omega, \phi^{2,1}$ is proportional to $\sin \left(\Theta_{\omega}(t)+\lambda\right), \lambda$ being the longitude, according to the Cartwright-Tayler convention. This is ensured by taking the spectral components of $\phi_{1}(t)$ and $\phi_{2}(t)$ to be

$\phi_{1}(t)=\phi\left(\sigma_{\mathrm{r}}\right) \cos \left(\Theta_{\omega}-\pi / 2\right), \phi_{2}(t)=-\phi\left(\sigma_{\mathrm{r}}\right) \sin \left(\Theta_{\omega}-\pi / 2\right),(58)$

with $\phi\left(\sigma_{\mathrm{r}}\right)$ real and $-3 / 2<\sigma_{\mathrm{r}}<-1 / 2$. The complex quantities $\tilde{\phi}(t)$ and $\tilde{\phi}^{*}(t)$ are now

$$
\tilde{\phi}(t) \equiv \phi_{1}(t)+\mathrm{i} \phi_{2}(t)=\phi\left(\sigma_{\mathrm{r}}\right) \mathrm{e}^{-\mathrm{i}\left(\Theta_{\omega}-\pi / 2\right)} \quad \text { and }
$$

$\tilde{\phi}^{*}(t)=\phi\left(\sigma_{\mathrm{r}}\right) \mathrm{e}^{\mathrm{i}\left(\Theta_{\omega}-\pi / 2\right)}$.

The torque (57) becomes now

$\tilde{\Gamma}_{\omega}=\tilde{\Gamma}\left(\sigma_{\mathrm{r}}\right) \mathrm{e}^{-\mathrm{i}\left(\Theta_{\omega}(t)-\pi / 2\right)}+\tilde{\Gamma}\left(\sigma_{\mathrm{p}}\right) \mathrm{e}^{\mathrm{i}\left(\Theta_{\omega}-\pi / 2\right)}$,

$\tilde{\Gamma}\left(\sigma_{\mathrm{r}}\right)=-\mathrm{i} \bar{A} \Omega_{0}^{2} \phi\left(\sigma_{\mathrm{r}}\right) e, \quad \tilde{\Gamma}\left(\sigma_{\mathrm{p}}\right)=-\mathrm{i} \bar{A} \Omega_{0}^{2} \phi\left(\sigma_{\mathrm{r}}\right) Z$.

On using (60), the solution of Eqs. (54) is seen to have the form

$\tilde{m}(t)=\tilde{m}\left(\sigma_{\mathrm{r}}\right) \mathrm{e}^{-\mathrm{i}\left(\Theta_{\omega}-\pi / 2\right)}+\tilde{m}\left(\sigma_{\mathrm{p}}\right) \mathrm{e}^{\mathrm{i}\left(\Theta_{\omega}-\pi / 2\right)}$

together with a similar expression for $\tilde{m}_{\mathrm{f}}(t)$.

We can now specialize Eqs. (55) to the case of prograde wobbles by setting $\sigma=\sigma_{\mathrm{p}}$ and $\tilde{\Gamma}(\sigma)=\tilde{\Gamma}\left(\sigma_{\mathrm{p}}\right)$ and introducing from Eqs. (31) the expressions

$\tilde{c}_{3}\left(\sigma_{\mathrm{p}}\right)=\bar{A}\left[\kappa \tilde{m}\left(\sigma_{\mathrm{p}}\right)+\xi \tilde{m}_{\mathrm{f}}\left(\sigma_{\mathrm{p}}\right)\right]$,

$\tilde{c}_{3}^{\mathrm{f}}\left(\sigma_{\mathrm{p}}\right)=\bar{A}_{\mathrm{f}}\left[\gamma \tilde{m}\left(\sigma_{\mathrm{p}}\right)+\beta \tilde{m}_{\mathrm{f}}\left(\sigma_{\mathrm{p}}\right)\right]$,

noting that $\tilde{\phi}\left(\sigma_{\mathrm{p}}\right)=0$ since the potential is strictly retrograde. We thus obtain the wobble equations for $\sigma=\sigma_{\mathrm{p}}$ in explicit form as

$\left[\left(\sigma_{\mathrm{p}}-e\right)+\left(1+\sigma_{\mathrm{p}}\right) \kappa\right] \tilde{m}\left(\sigma_{\mathrm{p}}\right)+\left(1+\sigma_{\mathrm{p}}\right)\left(\bar{A}_{\mathrm{f}} / \bar{A}+\xi\right) \tilde{m}_{\mathrm{f}}\left(\sigma_{\mathrm{p}}\right)$

$=-Z \phi\left(\sigma_{\mathrm{r}}\right)+\left(1+\sigma_{\mathrm{p}}\right)\left[Z \tilde{m}^{*}\left(\sigma_{\mathrm{r}}\right)+\left(\bar{A}_{\mathrm{f}} / \bar{A}\right) Z_{\mathrm{f}} \tilde{m}_{\mathrm{f}}^{*}\left(\sigma_{\mathrm{r}}\right)\right]$,

$(1+\gamma) \sigma_{\mathrm{p}} \tilde{m}\left(\sigma_{\mathrm{p}}\right)+\left[1+(1+\beta) \sigma_{\mathrm{p}}+e_{\mathrm{f}}\right] \tilde{m}_{\mathrm{f}}\left(\sigma_{\mathrm{p}}\right)$

$=Z_{\mathrm{f}} \sigma_{\mathrm{p}} \tilde{m}^{*}\left(\sigma_{\mathrm{r}}\right)+Z_{\mathrm{f}} \sigma_{\mathrm{p}} \tilde{m}_{\mathrm{f}}^{*}\left(\sigma_{\mathrm{r}}\right)$.

Now, $\tilde{m}^{*}\left(\sigma_{\mathrm{r}}\right)$ and $\tilde{m}_{\mathrm{f}}^{*}\left(\sigma_{\mathrm{r}}\right)$ relate to the retrograde diurnal wobbles. The triaxiality parts of the angular momenta of the Earth and its core, which are the source of the terms containing these quantities in the above equations, are thus seen to couple the prograde diurnal wobbles to the retrograde ones. Thus the mechanism through which the resonances in the latter get to affect the prograde semidiurnal nutations becomes transparent.

In the coefficients of $\tilde{m}\left(\sigma_{\mathrm{p}}\right)$ and $\tilde{m}_{\mathrm{f}}\left(\sigma_{\mathrm{p}}\right)$ in Eqs. $(64), \sigma_{\mathrm{p}}$ is close to 1 while all other quantities except $A_{\mathrm{f}} / A$ are of $O(\epsilon)$. Substituting for $\left(1+\sigma_{\mathrm{p}}\right) \tilde{m}_{\mathrm{f}}\left(\sigma_{\mathrm{p}}\right)$ in Eqs. (64a) from (64b), one finds, with the neglect of small quantities of $O(\epsilon)$, that

$$
\begin{aligned}
\left(\bar{A}_{\mathrm{m}} / \bar{A}\right) \sigma_{\mathrm{p}} \tilde{m}\left(\sigma_{\mathrm{p}}\right)= & -Z \tilde{\phi}\left(\sigma_{\mathrm{r}}\right)+\left(1+\sigma_{\mathrm{p}}\right) Z \tilde{m}^{*}\left(\sigma_{\mathrm{r}}\right) \\
& +\left(\overline{A_{\mathrm{f}}} / \bar{A}\right) Z_{\mathrm{f}}\left[\tilde{m}_{\mathrm{f}}^{*}\left(\sigma_{\mathrm{r}}\right)-\sigma_{\mathrm{p}} \tilde{m}^{*}\left(\sigma_{\mathrm{r}}\right)\right] .
\end{aligned}
$$

This result enables us to compute the prograde semidiurnal nutations from the amplitudes of the LF nutations and of the associated wobbles of the core, since both $\tilde{\phi}\left(\sigma_{\mathrm{r}}\right)$ and $\tilde{m}\left(\sigma_{\mathrm{r}}\right)$ may be expressed in terms of the amplitude of the associated nutation with frequency $v=1+\sigma_{\mathrm{r}}$.

Before proceeding futher on these lines, it is useful to get an idea of the relative magnitudes of the various terms on the right hand side of (65). The dominant term is the first one representing the external torque acting on the triaxiality. Both $\tilde{m}^{*}\left(\sigma_{\mathrm{r}}\right)$ and $\tilde{m}_{\mathrm{f}}^{*}\left(\sigma_{\mathrm{r}}\right)$ in the remaining terms are affected by the resonance at $\sigma_{\mathrm{r}} \approx-1.002319$ associated with the FCN. Nevertheless, the factor $\tilde{m}^{*}\left(\sigma_{\mathrm{r}}\right) / \tilde{\phi}\left(\sigma_{\mathrm{r}}\right)$ stays close to $e$ at the frequencies of interest here, deviating from it only by about $30 \%$ even at the $\psi_{1}$ frequency $\left(\sigma_{\mathrm{r}}=-1.002730\right)$ responsible for the retrograde annual nutation. Therefore the contribution from the second term on the right in (65) relative to that of the first term is $\approx 2 e \approx 1 / 150$, since $\sigma_{\mathrm{p}}$ is close to unity; hence the contribution of this term to any of the semidiurnal polar motion coefficients is at most about $0.1 \mu$ as, the largest of the coefficients being about $15 \mu$ as. If the core is triaxial $\left(Z_{\mathrm{f}} \neq 0\right)$, the huge resonance in $\tilde{m}_{\mathrm{f}}^{*}\left(\sigma_{\mathrm{r}}\right)$ enters the picture: $\left|\tilde{m}_{\mathrm{f}}^{*}\left(\sigma_{\mathrm{r}}\right) / \tilde{m}^{*}\left(\sigma_{\mathrm{r}}\right)\right|$ is as large as 800 for the $\psi_{1}$ frequency, and about 200 at the frequency $(\sigma=-1)$ of the $K_{1}$ tide (which causes the precession) and at the nearby frequency with which the 18.6 year retrograde nutation is associated. Thus, if the triaxiality of the core were to have the same magnitude as that of the whole Earth $\left(Z_{\mathrm{f}}=Z\right)$, the magnitude of the contribution of the $\tilde{m}_{\mathrm{f}}^{*}\left(\sigma_{\mathrm{r}}\right)$ term in $(65)$ relative to that of the external torque would become $\left|\left(A_{\mathrm{f}} / A\right)\left(\tilde{m}_{\mathrm{f}}^{*}\left(\sigma_{\mathrm{r}}\right) / \tilde{m}^{*}\left(\sigma_{\mathrm{r}}\right)\right)\left(\tilde{m}^{*}\left(\sigma_{\mathrm{r}}\right) / \tilde{\phi}\left(\sigma_{\mathrm{r}}\right)\right)\right| \approx$ $(1 / 9)(200)(1 / 300) \approx 0.075$ for $\sigma_{\mathrm{p}}=1$ (the 0.99727 day polar motion driven by the $K_{1}$ tide); the corresponding number is large, about 0.39 , for the PM due to the $\psi_{1}$ tide close to the resonance, but only 0.0022 for that due to the $\mathrm{O}_{1}$ tide far from the resonance. The magnitude of the actual contribution to the larger of the PM coefficients for the 0.99727 day polar motion turns out to be about $1 \mu$ as, which is not insignificant. Further discussion of the numerical results on the effects of core triaxiality will be deferred to Sect. 8, where a comparison will be made with the results of Escapa et al. (2002) who have already drawn attention to the role of the FCN resonance in the context of semidiurnal nutations (equivalent to the prograde diurnal polar motions).

Returning now to the development of the theoretical expressions, we note that no generally accepted quantitative estimates are avaliable for the triaxiality of the core, and so we ignore its effects hereafter. We have then, to an approximation which neglects terms of $O(\epsilon)$,

$\tilde{m}\left(\sigma_{\mathrm{p}}\right)=-(Z / e)\left(\bar{A} / \bar{A}_{\mathrm{m}}\right) \tilde{m}_{\mathrm{R}}\left(\sigma_{\mathrm{r}}\right)$. 
In this approximation, the transfer function from the rigid to the nonrigid Earth is simply the constant $A / A_{\mathrm{m}}$.

Now, in view of Eq. (62), the prograde part of the wobble in the time domain is

$\tilde{m}(t)=\tilde{m}\left(\sigma_{\mathrm{p}}\right) \mathrm{e}^{\mathrm{i}\left(\Theta_{\omega}(t)-\pi / 2\right)}=-\mathrm{i} \tilde{m}\left(\sigma_{\mathrm{p}}\right) \mathrm{e}^{\mathrm{i} \Theta_{\omega}(t)}$.

The corresponding nutation is obtained using Eqs. (13), (15), and (16) with the upper sign, remembering that $m=1$ and $\mathrm{e}^{-\mathrm{i} \zeta_{n, m}}=-\mathrm{i}$ in the present case:

$\tilde{\eta}(t)=\frac{\mathrm{i} \tilde{m}\left(\sigma_{\mathrm{p}}\right)}{\left(\sigma_{\mathrm{p}}+1\right)} \mathrm{e}^{\mathrm{i}\left(\Theta_{\omega}(t)+G M S T\right)}=\mathrm{i} \tilde{\eta}(v) \mathrm{e}^{\mathrm{i} \Xi_{\nu}}$,

where $\tilde{\eta}(v)=-\tilde{m}\left(\sigma_{\mathrm{p}}\right) /\left(1+\sigma_{\mathrm{p}}\right)$ as in $(17)$, and $v=v_{+} \equiv 1+\sigma_{\mathrm{p}}$. As for $\tilde{p}(t)$, one finds from (19) on using (68) that

$\tilde{p}(t)=\frac{-\mathrm{i} \tilde{m}\left(\sigma_{\mathrm{p}}\right)}{\left(\sigma_{\mathrm{p}}+1\right)} \mathrm{e}^{\mathrm{i} \Theta_{\omega}(t)}$.

If we now use the approximation (66) for $\tilde{m}\left(\sigma_{\mathrm{p}}\right)$ and then replace $\tilde{m}_{\mathrm{R}}\left(\sigma_{\mathrm{r}}\right)$ by $-\left(1+\sigma_{\mathrm{r}}\right) \tilde{\eta}\left(v_{-}\right)$where $v_{-}=1-\sigma_{\mathrm{p}}$, and use the expression (50) for $Z$, we obtain

$\tilde{p}(t)=-\mathrm{i} \frac{2 M_{\mathrm{E}} a_{\mathrm{e}}^{2}}{\bar{A}_{\mathrm{m}} e} \frac{1+\sigma_{\mathrm{r}}}{1+\sigma_{\mathrm{p}}} \tilde{\eta}_{\mathrm{R}}\left(v_{-}\right)\left(C_{2,2}+\mathrm{i} S_{2,2}\right) \mathrm{e}^{\mathrm{i} \Theta_{\omega}(t)}$.

This expression may be evaluated by taking the amplitudes $\tilde{\eta}_{\mathrm{R}}\left(v_{-}\right)$of LF nutations from the appropriate rigid Earth nutation series. For exact results, one needs to compute the solution for $\tilde{m}\left(\sigma_{\mathrm{p}}\right)$ from Eqs. (64), which calls for a knowledge of $\tilde{m}\left(\sigma_{\mathrm{r}}\right)$ (and $\tilde{m}_{\mathrm{f}}\left(\sigma_{\mathrm{r}}\right)$ too if $Z_{\mathrm{f}}$ is set to a nonzero value). These wobble amplitudes may be obtained, for instance, by solving the standard Sasao et al. (1980) equations. It turns out that the largest of the errors caused by the use of (70) is at the $0.1 \mu$ as level if triaxiality of the core is ignored. The results shown in the tables in the next section are the exact ones, of course.

\section{Polar motion and nutation coefficients: Expressions and numerical results}

\subsection{Circular motions}

According to Eq. (20),

$\tilde{p}(t)=Q^{ \pm} \mathrm{e}^{ \pm \mathrm{i}_{\omega}(t)}$,

$Q^{+}=\tilde{p}\left(\sigma_{\mathrm{p}}\right) \mathrm{e}^{-\mathrm{i} \zeta_{n, m}}, \quad Q^{-}=\tilde{p}\left(\sigma_{\mathrm{r}}\right) \mathrm{e}^{\mathrm{i} \zeta_{n, m}}$.

Therefore, if we write $Q^{ \pm}=Q_{\mathrm{R}}^{ \pm}+\mathrm{i} Q_{\mathrm{I}}^{ \pm}$, it follows from Eq. (19) that the coefficients of $\sin \Theta_{\omega}(t)$ and $\cos \Theta_{\omega}(t)$ (identified by superscripts $\mathrm{s}$ and $\mathrm{c}$, respectively) in the polar motion variables are,

$x_{\mathrm{p}}^{\mathrm{s}}=\mp Q_{I}^{ \pm}, x_{\mathrm{p}}^{\mathrm{c}}=Q_{\mathrm{R}}^{ \pm}, y_{\mathrm{p}}^{\mathrm{s}}=\mp x_{\mathrm{p}}^{\mathrm{c}}=\mp Q_{\mathrm{R}}^{ \pm}, y_{\mathrm{p}}^{\mathrm{c}}= \pm x_{\mathrm{p}}^{\mathrm{s}}=-Q_{\mathrm{I}}^{ \pm}$.

As usual, the upper and lower signs are for prograde and retrograde circular polar motions (frequencies $\sigma_{\mathrm{p}}$ and $\sigma_{\mathrm{r}}=-\sigma_{\mathrm{p}}$, respectively), and $\omega$ and $\Theta_{\omega}(t)$ pertain to the tidal potential which excites these motions. The combined polar motion, which is elliptical, in general, is obtained by adding the two parts.
Table 3. Coefficients of $F_{n, m}^{( \pm)} \sin \Theta_{\omega}(t)$ and $F_{n, m}^{( \pm)} \cos \Theta_{\omega}(t)$ in polar motions excited by various potentials.

\begin{tabular}{|c|c|c|c|c|}
\hline \multirow{2}{*}{$\begin{array}{l}\text { Forcing } \\
\text { potential }\end{array}$} & \multicolumn{2}{|c|}{ Coefficients in $x_{\mathrm{p}}(t)$} & \multicolumn{2}{|c|}{ Coefficients in $y_{\mathrm{p}}(t)$} \\
\hline & $\sin \Theta_{\omega}(t)$ & $\cos \Theta_{\omega}(t)$ & $\sin \Theta_{\omega}(t)$ & $\cos \Theta_{\omega}(t)$ \\
\hline \multicolumn{5}{|c|}{ Prograde polar motions } \\
\hline$(2,1)$ & $C_{2,2}$ & $S_{2,2}$ & $-S_{2,2}$ & $C_{2,2}$ \\
\hline$(3,0)$ & $-C_{3,1}$ & $-S_{3,1}$ & $S_{3,1}$ & $-C_{3,1}$ \\
\hline$(3,1)$ & $-S_{3,2}$ & $C_{3,2}$ & $-C_{3,2}$ & $-S_{3,2}$ \\
\hline$(3,2)$ & $-C_{3,3}$ & $-S_{3,3}$ & $S_{3,3}$ & $-C_{3,3}$ \\
\hline$(4,0)$ & $S_{4,1}$ & $-C_{4,1}$ & $C_{4,1}$ & $S_{4,1}$ \\
\hline$(4,1)$ & $C_{4,2}$ & $S_{4,2}$ & $-S_{4,2}$ & $C_{4,2}$ \\
\hline \multicolumn{5}{|c|}{ Retrograde polar motions } \\
\hline$(3,0)$ & $-C_{3,1}$ & $S_{3,1}$ & $S_{3,1}$ & $C_{3,1}$ \\
\hline$(3,2)$ & $C_{3,1}$ & $S_{3,1}$ & $S_{3,1}$ & $-C_{3,1}$ \\
\hline$(3,3)$ & $S_{3,2}$ & $-C_{3,2}$ & $-C_{3,2}$ & $-S_{3,2}$ \\
\hline$(4,0)$ & $-S_{4,1}$ & $-C_{4,1}$ & $-C_{4,1}$ & $S_{4,1}$ \\
\hline
\end{tabular}

The explicit expressions for $\tilde{p}(\sigma)$ which have to be used for computing the above coefficients follow from Eq. (37) taken together with (26a), (26b), or (26c), as the case may be:

$$
\begin{aligned}
Q^{ \pm} & =F_{n, m}^{( \pm)}\left[(-1)^{m+1} \mathrm{e}^{\mp \mathrm{i} \zeta_{n, m}}\left( \pm C_{n, m \pm 1}+\mathrm{i} S_{n, m \pm 1}\right)\right], \\
F_{n, m}^{( \pm)} & =\frac{A}{A_{\mathrm{m}}} \frac{1}{\left(1 \pm \sigma_{\mathrm{p}}\right)\left( \pm \sigma_{\mathrm{p}}-\sigma_{1}\right)} G_{n, m}^{( \pm)} H_{\omega}^{(n, m)},
\end{aligned}
$$

with the exception that for the retrograde case with $m=0$,

$$
\begin{aligned}
Q^{-} & =F_{n, 0}^{(-)}\left[-\mathrm{e}^{\mathrm{i} \zeta n, 0}\left(C_{n, 1}+\mathrm{i} S_{n, 1}\right)\right] \\
F_{n, 0}^{(-)} & =\frac{A}{A_{\mathrm{m}}} \frac{1}{\left(1-\sigma_{\mathrm{p}}\right)\left(-\sigma_{\mathrm{p}}-\sigma_{1}\right)} G_{n, 0}^{(+)} H_{\omega}^{(n, 0)}
\end{aligned}
$$

The real part of the square bracketed factor is, in general, one of the pair of geopotential coefficients ( $C$ or $S$ ) of the relevant degree and order, and the imaginary part is the other member of the pair, both to within a sign; what sign goes with each, is determined by the parities (odd/even) of $m$ and $(n-m)$. In view of Eqs. (73), the coefficients of polar motion are now directly given by these quantities, apart from the factor $F_{n, m}^{( \pm)}$, as shown in Table 3, provided that $F_{n, m}^{( \pm)}$can be taken to be real. The reality assumption is not strictly valid because of the presence of an imaginary part in the Chandler frequency $\sigma_{1}$, but its effect is quite negligible except for excitation frequencies close to $\sigma_{1}$, which exist only in the case of $(n, 0)$ potentials. Close to the resonance, the sine and cosine coefficients contain admixtures of $C_{n, 1}$ and $S_{n, 1}$, weighted by the real and imaginary parts of $F_{n, 0}^{( \pm)}$.

We have carried out the numerical evaluation of the coefficients of polar motions due to tidal potentials of degrees up to 4 , starting from the tidal amplitudes defined according to the conventions of Cartwright \& Tayler (1971). Actually, we used the RATGP series of Roosbeek (1996) and converted the amplitudes from this series to their Cartwright-Tayler equivalents through multiplication by the appropriate factors $f_{n, m}$ taken from Table 6.5 of the IERS Conventions 1996. (The sign of the factor $f_{3,1}$ given there has to be reversed; the value $f_{4,0}=0.317600$ not listed there was needed to compute polar motions with coefficients down to $0.05 \mu$ as, there being a 
few that are excited by $(4,0)$ potentials.) For the prograde diurnal polar motions, we have also used the alternative approach explained in Sect. 7, using the known amplitudes of the long period nutations as inputs instead of the tidal amplitudes. The JGM3 values listed in Table 2 were used for the geopotential coefficients in computations for the nonrigid Earth; the IERS92 values were used for the rigid Earth case, to facilitate comparisons with the results of earlier workers.

We present in Table 4 the periodic polar motions having amplitudes exceeding $0.5 \mu$ as. Only the low frequency polar motions due to $(3,0)$ potentials and the prograde diurnals excited by the $(2,1)$ potentials attain these magnitudes. The secular polar motion due to the constant term in the $(4,0)$ potential is also shown. The argument of the polar motion, denoted by ( $\arg )$ in the Table, is $\Theta_{\omega}(t)$ if the motion is prograde, and $-\Theta_{\omega}(t)$ if retrograde. It is expressed here as a linear combination,

$(\arg )=n_{1}(\Phi+\pi)+n_{2} l+n_{3} l^{\prime}+n_{4} F+n_{5} D+n_{6} \Omega$,

where $\Phi=G M S T$ and $l, l^{\prime}, F, D, \Omega$, (which are themselves linear combinations of the last five of Doodson's tidal arguments) are DeLaunay's fundamental arguments for nutations. For polar motions excited by potentials of type $(n, m), n_{1}= \pm m$ according as the motion is prograde/retrograde. Retrograde motions do not appear in Table 4, as their amplitudes are below the cut-off, except for those excited by $(2,1)$ potentials which, however, are treated as nutations rather than polar motions.

The argument of the HF nutation equivalent to the polar motion of (76) is

$\left(n_{1}+1\right) \Phi+n_{2} l+n_{3} l^{\prime}+n_{4} F+n_{5} D+n_{6} \Omega$.

The periods of these circular nutations are shown in the last column of the table for convenience. They are all prograde.

\subsection{Coefficents of circular high frequency nutations}

The coefficients of the HF nutations may be inferred from those of the equivalent polar motions from the following considerations.

Beginning with the fact that $\tilde{\eta}(t)=-\tilde{p}(t) \mathrm{e}^{\mathrm{i} G M S T}$, which produces an overall sign difference between the coefficients of $\sin \Theta_{\omega}(t)$ and $\cos \Theta_{\omega}(t)$ in $x_{\mathrm{p}}(t)$ and $y_{\mathrm{p}}(t)$, on the one hand, and those of $\sin \Xi_{v}(t)$ and $\cos \Xi_{v}(t)$ in $\Delta \psi(t) \sin \epsilon$ and $\Delta \epsilon$ on the other hand, one takes note of the other sign differences that occur: a sign $(-1)^{m}$ depending on the order $m$ of the tidal potential giving rise to the motions, which arises from the relations (15) between $\Theta_{\omega}(t)$ and $\Xi_{v}(t)$, and a further minus sign that arises between the coefficients in $y_{\mathrm{p}}(t)$ on the one hand and those in $\Delta \epsilon(t)$ on the other because of the fact that $\tilde{p}(t)=x_{\mathrm{p}}(t)-\mathrm{i} y_{\mathrm{p}}(t)$ while $\tilde{\eta}(t)=\Delta \psi \sin \epsilon+\mathrm{i} \Delta \epsilon$. Thus, with superscripts $s$ and $c$ identifying coefficients of the sine and cosine functions, respectively, of ( $\arg )$ or $\Xi_{v}$ as the case may be, we have

$\Delta \psi_{v}^{\mathrm{s}} \sin \epsilon=(-1)^{m+1} x_{\mathrm{p}}^{\mathrm{s}}(\sigma), \quad \Delta \psi_{v}^{\mathrm{c}} \sin \epsilon=(-1)^{m+1} x_{\mathrm{p}}^{\mathrm{c}}(\sigma)$,

$\Delta \epsilon_{v}^{\mathrm{s}}=(-1)^{m} y_{\mathrm{p}}^{\mathrm{s}}(\sigma) \quad \Delta \epsilon_{v}^{\mathrm{c}}=(-1)^{m} y_{\mathrm{p}}^{\mathrm{c}}(\sigma)$.

The coefficients thus obtained are for circular nutations.

The above relations, when combined with (73), show that the coeffients of any circular polar motion and of the corresponding nutation can all be obtained from just two of them, say $x_{\mathrm{p}}^{\mathrm{s}}$ and $x_{\mathrm{p}}^{\mathrm{c}}$. They supersede Eqs. (24) of Mathews \& Bretagnon (2002) which fail to be valid in general as the sign factors referred to below Eq. (17) were overlooked.

Our results for a few of the leading terms in the semidiurnal nutations due to degree 2 potentials, which are strictly prograde and hence circular, are compared with the results from earlier works in Table 5. The coefficients $\Delta \epsilon^{\mathrm{s}}$ and $\Delta \epsilon^{\mathrm{c}}$ are shown both for the rigid and the nonrigid Earth; $\Delta \psi^{\mathrm{s}}=-\Delta \epsilon^{\mathrm{c}} / \sin \epsilon_{0}$ and $\Delta \psi^{\mathrm{c}}=\Delta \epsilon^{\mathrm{s}} / \sin \epsilon_{0}$ in the present case. The values shown against $\mathrm{BCpc}$ were obtained by conversion from recent polar motion coefficients of Brzeziński \& Capitaine (private communication, 2002). Elliptical nutations (including semidiurnal ones) that are induced by higher degree potentials are considered below.

\subsection{Elliptical motions}

The combination of two circular motions differing only in the sign of the frequency describes an elliptical motion. In Table 4, such prograde-retrograde pairs of terms appear only among the low frequency polar motions. The argument $\Theta_{\omega}(t)$ of the prograde part is assigned to the elliptical polar motion. Since $(\arg )=-\Theta_{\omega}(t)$ for the retrograde part, the signs of the coefficients in the sine columns have to be reversed in the row pertaining to any retrograde PM before adding to the coefficients in the row pertaining to the corresponding prograde PM to obtain the coefficients for the elliptical motion. For the elliptical PM with the 27.322 day period, for instance, one finds the coefficients (in the same order as in the table) to be $(0.89,3.99,-0.11,32.35) \mu$ as; they are $(-28.49,-0.24,3.44,-3.85) \mu$ as for the 3231.496 day polar motion. Note the predominance of the cosine part of $y_{\mathrm{p}}$ in the former case and of the sine part of $x_{\mathrm{p}}$ in the latter. The difference in behaviour is due to the presence of the Chandler mode in between these periods.

For higher frequency PM, (e.g., the semidiurnals), the prograde and retrograde parts originate in the action of the same potential on different geopotential coefficients, e.g., by the action of $(3,2)$ potentials on $C_{3,3}$ and $S_{3,3}$ for prograde semidiurnals, and on $C_{3,1}$ and $S_{3,1}$ for the retrograde ones. The largest of these, with periods of +0.52752 and -0.52752 have coefficients $(-0.330,-.0041,-0.041,0.330)$ and $(-0.028,-0.055,0.055,-0.028) \mu \mathrm{as}$, respectively; both sets are below the cuf-off for inclusion in Table 4. The elliptical motion from their combination has coefficients $(-0.358,-0.096,0.014,0.302) \mu$ as.

In contrast to elliptical polar motions, elliptical HF nutations result from the combination of a pair of prograde and retrograde nutations produced by different potentials acting on the same $C$ and $S$ coefficients. It must be noted that the semidiurnal nutations arising from the action of degree 2 potentials on $C_{2,2}$ and $S_{2,2}$ are strictly prograde and circular: there exists no (2, $3)$ potential to generate retrograde components. Thus the elliptical nutations are generated only by higher degree potentials. Table 6 shows a few examples from our computations for the rigid Earth, and comparisons with the results of Bretagnon et al. (1997) and Folgueira et al. (2001) - both of which are for elliptical nutations only. Since both these works have employed 
Table 4. Coefficients, in $\mu$ as, of $\sin (\arg )$ and $\cos (\arg )$, $\arg = \pm \Theta_{\omega}(t)$, in polar motions $\left(x_{\mathrm{p}}(t), y_{\mathrm{p}}(t)\right)$ excited by potentials of different degrees $n$, and periods, in solar days, of equivalent high frequency nutations.

\begin{tabular}{|c|c|c|c|c|c|c|c|c|c|c|c|c|}
\hline \multirow[t]{2}{*}{$n$} & \multicolumn{6}{|c|}{ Multipliers of } & \multirow{2}{*}{$\begin{array}{l}\text { Period } \\
\text { of PM }\end{array}$} & \multicolumn{2}{|c|}{$x_{\mathrm{p}}(t)$} & \multicolumn{2}{|c|}{$y_{\mathrm{p}}(t)$} & \multirow{2}{*}{$\begin{array}{r}\text { Nutation } \\
\text { Period }\end{array}$} \\
\hline & $\Phi+\pi$ & $l$ & $l^{\prime}$ & $F$ & $D$ & $\Omega$ & & $\sin$ & $\cos$ & $\sin$ & $\cos$ & \\
\hline 3 & 0 & -1 & 0 & -1 & 0 & -1 & -13.719 & 1.39 & .17 & -.17 & 1.39 & 1.07545 \\
\hline 3 & 0 & 0 & 0 & -1 & 0 & 0 & -27.212 & 2.48 & .30 & -.30 & 2.48 & 1.03521 \\
\hline 3 & 0 & 0 & 0 & -1 & 0 & -1 & -27.322 & 15.75 & 1.93 & -1.93 & 15.75 & 1.03505 \\
\hline 3 & 0 & 0 & 0 & -1 & 0 & -2 & -27.432 & -.82 & -.10 & .10 & -.82 & 1.03489 \\
\hline 3 & 0 & -1 & 0 & -1 & 2 & -1 & -193.560 & .81 & .10 & -.10 & .81 & 1.00243 \\
\hline 3 & 0 & 1 & 0 & -1 & 0 & 0 & -2190.35 & 1.86 & .24 & -.24 & 1.86 & .99772 \\
\hline 3 & 0 & 1 & 0 & -1 & 0 & -1 & -3231.50 & 12.32 & 1.59 & -1.59 & 12.32 & .99758 \\
\hline 3 & 0 & 1 & 0 & -1 & 0 & -2 & -6159.14 & -.68 & -.09 & .09 & -.68 & .99743 \\
\hline 3 & 0 & -1 & 0 & 1 & 0 & 2 & 6159.14 & .78 & .09 & -.09 & .78 & .99711 \\
\hline 3 & 0 & -1 & 0 & 1 & 0 & 1 & 3231.50 & -16.16 & -1.83 & 1.83 & -16.16 & .99696 \\
\hline 3 & 0 & -1 & 0 & 1 & 0 & 0 & 2190.35 & -2.78 & -.31 & .31 & -2.78 & .99682 \\
\hline 3 & 0 & 1 & 1 & -1 & 0 & 0 & 438.360 & -.63 & .12 & -.12 & -.63 & .99501 \\
\hline 3 & 0 & 1 & 1 & -1 & 0 & -1 & 411.807 & 1.05 & .27 & -.27 & 1.05 & .99486 \\
\hline 3 & 0 & 0 & 0 & 1 & -1 & 1 & 365.242 & 1.31 & .20 & -.20 & 1.31 & .99455 \\
\hline 3 & 0 & 1 & 0 & 1 & -2 & 1 & 193.560 & 2.10 & .27 & -.27 & 2.10 & .99216 \\
\hline 3 & 0 & 0 & 0 & 1 & 0 & 2 & 27.432 & -.87 & -.11 & .11 & -.87 & .96229 \\
\hline 3 & 0 & 0 & 0 & 1 & 0 & 1 & 27.322 & 16.64 & 2.04 & -2.04 & 16.64 & .96215 \\
\hline 3 & 0 & 0 & 0 & 1 & 0 & 0 & 27.212 & 2.62 & .32 & -.32 & 2.62 & .96201 \\
\hline 3 & 0 & 1 & 0 & 1 & 0 & 1 & 13.719 & 1.28 & .16 & -.16 & 1.28 & .92969 \\
\hline 2 & 1 & -1 & 0 & -2 & 0 & -1 & 1.11970 & -.44 & .25 & -.25 & -.44 & .52747 \\
\hline 2 & 1 & -1 & 0 & -2 & 0 & -2 & 1.11951 & -2.31 & 1.32 & -1.32 & -2.31 & .52743 \\
\hline 2 & 1 & 1 & 0 & -2 & -2 & -2 & 1.11346 & -.44 & .25 & -.25 & -.44 & .52608 \\
\hline 2 & 1 & 0 & 0 & -2 & 0 & -1 & 1.07598 & -2.14 & 1.23 & -1.23 & -2.14 & .51756 \\
\hline 2 & 1 & 0 & 0 & -2 & 0 & -2 & 1.07581 & -11.36 & 6.52 & -6.52 & -11.36 & .51753 \\
\hline 2 & 1 & -1 & 0 & 0 & 0 & 0 & 1.03472 & .84 & -.48 & .48 & .84 & .50782 \\
\hline 2 & 1 & 0 & 0 & -2 & 2 & -2 & 1.00275 & -4.76 & 2.73 & -2.73 & -4.76 & .50000 \\
\hline 2 & 1 & 0 & 0 & 0 & 0 & 0 & .99727 & 14.27 & -8.19 & 8.19 & 14.27 & .49863 \\
\hline 2 & 1 & 0 & 0 & 0 & 0 & -1 & .99712 & 1.93 & -1.11 & 1.11 & 1.93 & .49860 \\
\hline 2 & 1 & 1 & 0 & 0 & 0 & 0 & .96244 & .76 & -.43 & .43 & .76 & .48977 \\
\hline
\end{tabular}

Rate of secular polar motion ( $\mu$ as/yr) due to the zero frequency tide, $\arg =0$

\begin{tabular}{llllllllll}
4 & 0 & 0 & 0 & 0 & 0 & 0 & -3.80 & -4.31 & .99727 \\
\hline
\end{tabular}

Table 5. Coefficients (in $\mu$ as) of prograde semidiurnal nutations: Comparisons with earlier works ${ }^{a}$.

\begin{tabular}{rrccccr}
\hline \hline $\begin{array}{r}\text { Period } \\
\text { (days) }\end{array}$ & Authors & $\begin{array}{c}\text { Rigid Earth } \\
\Delta \epsilon^{\mathrm{s}}\end{array}$ & $\Delta \epsilon^{\mathrm{c}}$ & Authors & $\Delta \epsilon^{\mathrm{s}}$ & $\Delta \epsilon^{\mathrm{c}}$ \\
\hline \multirow{2}{*}{0.51753} & BRS97 & 5.79 & 10.09 & GFE01 & 6.54 & 11.39 \\
& FBS01 & 5.8 & 10.0 & FBS01 & 6.5 & 11.3 \\
& BCpc & 5.83 & 10.16 & BCpc & 6.57 & 11.45 \\
& Present & 5.87 & 10.22 & Present & 6.52 & 11.36 \\
\hline 0.50000 & BRS97 & 2.43 & 4.23 & GFE01 & 2.74 & 4.77 \\
& FBS01 & 2.37 & 4.13 & FBS01 & 2.7 & 4.7 \\
& BCpc & 2.44 & 4.25 & BCpc & 2.75 & 4.79 \\
& Present & 2.46 & 4.28 & Present & 2.73 & 4.76 \\
\hline \multirow{2}{*}{0.49863} & BRS97 & -7.27 & -12.67 & GFE01 & -8.21 & -14.30 \\
& FBS01 & -7.12 & -12.40 & FBS01 & -8.0 & -14.0 \\
& BCpc & -7.32 & -12.75 & BCpc & -8.25 & -14.37 \\
& Present & -7.27 & -12.67 & Present & -8.19 & -14.27 \\
\hline
\end{tabular}

${ }^{a}$ BRS97: Bretagnon et al. (1997); FBS01: Folgueira et al. (2001); GFE01: Getino et al. (2001); and BCpc: Brzeziński \& Capitaine (private communication, 2002). 
Table 6. Coefficients (in $\mu$ as) of elliptical nutations of the rigid Earth; Comparisons with Bretagnon et al. (1997) and Folgueira et al. (2001) (abbreviated as BRS97 and FBS01).

\begin{tabular}{|c|c|c|c|c|c|c|}
\hline \multirow{2}{*}{$\begin{array}{r}\text { Type of } \\
\text { Tide }\end{array}$} & \multicolumn{2}{|c|}{ Period (days) of } & \multicolumn{4}{|c|}{ Coefficients } \\
\hline & Wobble & Nutation & $\Delta \psi^{\mathrm{s}}$ & $\Delta \psi^{\mathrm{c}}$ & $\Delta \epsilon^{\mathrm{s}}$ & $\Delta \epsilon^{\mathrm{c}}$ \\
\hline \multicolumn{7}{|c|}{ Diurnal nutations } \\
\hline$(3,0)$ & -27.322 & 1.03505 & -34.201 & -4.204 & -1.672 & 13.604 \\
\hline$(3,2)$ & -.50790 & -1.03505 & .604 & -.074 & -.030 & -.240 \\
\hline Total & & & -34.805 & -4.278 & -1.642 & 13.364 \\
\hline BRS97 & & & -34.821 & -4.271 & -1.640 & 13.371 \\
\hline FBS01 & & & -35.404 & -4.351 & -1.587 & 12.911 \\
\hline$(3,0)$ & -3231.5 & .99758 & -19.881 & -2.444 & -.972 & 7.908 \\
\hline$(3,2)$ & -.49871 & -.99758 & .031 & -.004 & -.002 & -.012 \\
\hline Total & & & -19.912 & -2.448 & -.970 & 7.896 \\
\hline BRS97 & & & -19.854 & -2.491 & -.988 & 7.873 \\
\hline FBS01 & & & -19.940 & -2.451 & -.972 & 7.906 \\
\hline$(3,0)$ & 27.322 & .96215 & -38.080 & -4.680 & -1.862 & 15.147 \\
\hline$(3,2)$ & -.48970 & -.96215 & .050 & -.006 & -.002 & -.020 \\
\hline Total & & & $-38,130$ & -4.686 & -1.860 & 15.127 \\
\hline BRS97 & & & -38.128 & -4.695 & -1.863 & 15.127 \\
\hline FBS01 & & & -38.231 & -4.699 & -1.857 & 15.106 \\
\hline \multicolumn{7}{|c|}{ Semidiurnal nutations } \\
\hline$(3,1)$ & .89050 & .527517 & -.074 & -.108 & -.043 & .029 \\
\hline$(3,3)$ & -2.89050 & -.527517 & .106 & -.154 & -.061 & -.042 \\
\hline Total & & & -.180 & -.262 & .018 & -.013 \\
\hline BRS97 & & & -.178 & -.258 & .020 & -.013 \\
\hline FBS01 & & & -.109 & -.388 & .234 & -.092 \\
\hline$(3,1)$ & .963499 & .507904 & -.206 & -.301 & -.120 & .082 \\
\hline$(3,3)$ & -2.963499 & -.507904 & .013 & -.019 & -.008 & -.005 \\
\hline Total & & & -.219 & -.320 & -.112 & .077 \\
\hline BRS97 & & & -.219 & -.321 & -.113 & .077 \\
\hline FBS01 & & & -.244 & -.356 & -.097 & .067 \\
\hline
\end{tabular}

the values listed under IERS92 in Table 2 for $C_{n, m}$ and $S_{n, m}$, our numbers used for the comparison are based on the same values. The ratios $\left(-\Delta \psi^{\mathrm{s}} / \Delta \psi^{\mathrm{c}}\right)$ and $\left(\Delta \epsilon^{\mathrm{c}} / \Delta \epsilon^{\mathrm{s}}\right)$ should be equal to $\left(-C_{3,1} / S_{3,1}\right)$ for the diurnal nutations and $\left(S_{3,2} / C_{3,2}\right)$ for the semidiurnals, as may be seen from our theory. This requirement is satisfied rather well by all the sets of coefficients shown, except those of Folgueira et al. (2001) for the 0.527517 day nutation which are inconsistent with the above requirement. In fact, the fractional differences of their numbers from ours are not really small for the other listed semidiurnals too. Our sets of values are very close to those of Bretagnon et al.; and for the diurnal nutations, they are quite close to Folgueira et al. too.

\subsection{Effect of triaxiality of the core}

The coefficients shown in Table 4 for the prograde diurnal polar motions do not take account of possible triaxiality of the core. How much of a difference could core triaxiality make? To answer this question, we have made computations based on Eqs. (64) with nonzero $Z_{\mathrm{f}}$ as well as with $Z_{\mathrm{f}}=0$, and taken the difference. To facilitate comparison with the results of Escapa et al. (2002), we present in Table 7 our results for the contributions from $Z_{\mathrm{f}}$ to the equivalent semidiurnal nutations when $Z_{\mathrm{f}}=0.8112 Z$, together with numbers from the IT columns of Table 1 of their paper which pertain to the same ratio for $Z_{\mathrm{f}} / Z$ which is, in their notation, $d_{\mathrm{c}} / d$. Only the coefficients of the increment $\delta \Delta \epsilon$ due to $Z_{\mathrm{f}}$ are shown. It is evident that the Escapa et al. values are 2.4 to 3 times as large as ours, except for the .51753 day nutation for which the factor is nearly 8 . We have not been able to discern the reason for the discrepancies; and we find no scope for modifying our expressions to bridge the gap, our derivations being entirely transparent.

\section{Concluding remarks}

The comparisons presented in the last section show that the coefficients of high frequency nutations or of corresponding polar motions, as presented by different groups, do not differ by more than a few tenths of a microarcsecond for any of the frequencies involved. This is not surprising, considering that the largest of the polar motion amplitudes are under $20 \mu \mathrm{as}$. We focus here, therefore, on a few special features referred to in earlier sections. 
Table 7. Contributions (in $\mu$ as) from triaxiality of the core (with $Z_{\mathrm{f}}=$ $0.8112 Z$ ) to coefficients of semidiurnal nutations; comparison with Escapa et al. (2002).

\begin{tabular}{rrrrrr}
\hline \hline \multicolumn{2}{c}{ Nutation period ${ }^{a}$} & \multicolumn{2}{c}{ Present work } & \multicolumn{2}{c}{ Escapa et al. } \\
PSD & LF & $\delta \Delta \epsilon^{\mathrm{s}}$ & $\delta \Delta \epsilon^{\mathrm{c}}$ & $\delta \Delta \epsilon^{\mathrm{s}}$ & $\delta \Delta \epsilon^{\mathrm{c}}$ \\
\hline .49863 & $\infty$ & .468 & .815 & 1.211 & 2.110 \\
.49860 & -6798.38 & .068 & .118 & .175 & .304 \\
.50000 & 182.62 & -.045 & -.079 & -.134 & -.233 \\
.49795 & -365.26 & -.021 & -.036 & -.049 & -.085 \\
.51753 & 13.66 & -.010 & -.017 & -.076 & -.132 \\
\hline
\end{tabular}

${ }^{a}$ Periods, in solar days, of the prograde semidiurnal (PSD) and low frequency (LF) nutations produced by the same retrograde diurnal potential are shown in each row.

Firstly, the Chandler resonance in the low frequency polar motions: We pointed out at the beginning of Sect. 6 that the resonance frequency varies with the frequency of excitation. We find that the largest effect of this variation is on the \pm 3231 day polar motions: -0.08 and $0.16 \mu$ as, respectively, on the coefficients $x_{\mathrm{p}}^{\mathrm{s}}$ and $x_{\mathrm{p}}^{\mathrm{c}}$ of the retrograde one, and -0.17 and 0.02 on those of the prograde one. These are not ignorable at the $0.1 \mu$ as level. Another point concerns the flipping of the sign of the imaginary part of the resonance frequency which has to accompany the passage from positive to negative excitation frequencies. If this flip were ignored, $\left(x_{\mathrm{p}}^{\mathrm{s}}, x_{\mathrm{p}}^{\mathrm{c}}\right)$ would be in error by $(-0.17,0.02) \mu$ as for the -3231 day polar motion.

Secondly, the presence of the secular term in the polar motion of the nonrigid Earth due to the constant part of the $(4,0)$ potential (Sect. 6 and Table 4), which was noted already by Mathews \& Bretagnon (2002) and Brzeziński \& Capitaine (2002): It seems necessary to point out here that the "observed secular motion of the pole", referred to by the latter, is a linear drift of the Earth's rotation pole in relation to the TRF while the polar motions dealt with here (and elsewhere) in the context of the transformation between the CRF and the TRF are the secular and periodic motions (with periods outside the retrograde diurnal band) of the pole of the $C R F$ as seen from the TRF. It is unfortunate that the use of the term "polar motion" with different meanings in different contexts lends scope for avoidable confusion.

Thirdly, the effects of possible triaxiality $Z_{\mathrm{f}}$ of the core: We have brought out explicitly the coupling of the retrograde and prograde diurnal wobbles that is produced by the $Z$ and $Z_{\mathrm{f}}$ terms in the angular momentum, and the role played thereby in the prograde semidiurnal nutations by the FCN resonance (more specifically, by the resonance in the retrograde diurnal wobble of the core) through this coupling. We find the increments to the wobble eigenfrequencies due to triaxiality to be of no observational consequence: they are of the second order in the triaxiality parameter, proportional to $e^{\prime 2} \equiv Z Z^{*}$ (as noted in the first para of Sect. 7.3) or to $e_{\mathrm{f}}^{\prime 2} \equiv Z_{\mathrm{f}} Z_{\mathrm{f}}^{*}$, as the case may be, in contrast to the first order changes found by Escapa et al. (2002). (It is hard to comprehend how their first order changes could involve the moduli of the triaxiality parametes rather than the complex parameters themselves.) In regard to the forced motions, we have confirmed that the contributions from core triaxiality $Z_{\mathrm{f}}$ could be significant, as observed by Escapa et al. especially if $Z_{\mathrm{f}}$ were a few times $Z$; but the numbers we obtain for the these contributions are only about $40 \%$ of those reported by these authors, or less. Brzezińsky \& Capitaine (2002) have computed $Z_{\mathrm{f}}$ from the mantle tomographic model of Morelli \& Dziewonski (1987) and the mantle convection model of Defraigne et al. (1996). But the modeling of the core mantle boundary with the precision needed to make a useful estimate of $Z_{\mathrm{f}}$ is notoriously difficult, and the general consensus seems to be that the value of $Z_{\mathrm{f}}$ remains highy uncertain. One might perhaps hope that, if the core triaxiality were large enough, its estimation from observations of diurnal polar motions/semidiurnal nutations would become possible if and when the precision of estimation of such motions approaches the $1 \mu$ as level. However, it is important to take note that the prograde diurnal polar motions considered here, which are due to the torques exerted on the triaxial structure by degree 2 tesseral $(m=1)$ tidal potentials, are at least 10 times smaller than those due to the ocean tides raised by the very same tidal potentials; see Table 6 of Chao et al. (1996) for a comparative listing of observational estimates and theoretical predictions from various works. Even if the contribution from $Z_{\mathrm{f}}$, which is largest for the nutation period of 0.49863 days ( 0.99727 day polar motion), were as large as a few $\mu$ as, it would still be only a few percent of the ocean tide contribution for the same period. To estimate the core triaxiality contribution accurately enough from observations on prograde diurnal polar motions to permit useful bounds to be placed on the triaxiality $Z_{\mathrm{f}}$, one needs predictions for the dominant ocean tide contribution that are good at least at the $1 \mu$ as level. The prospects for modeling the ocean tide contribution to this level of accuracy seem quite dim, given the uncertainties in the modeling of ocean tides and their effects.

Acknowledgements. We are happy to acknowledge illuminating discussions with Aleksander Brzezińsky during the final stages of the preparation of this paper. We are also indebted to an anonymous referee for a detailed and thoughtful review of the paper, leading to improvements in presentation.

\section{Appendix A: Torque due to the potential of type $(n, m)$}

We start with the expression (4), written slightly more explicitly:

$$
\begin{aligned}
V_{\omega}^{(n, m)}(\boldsymbol{r} ; t)= & \frac{1}{2}\left(\frac{r}{a_{\mathrm{e}}}\right)^{n} \sum_{\omega} g_{\mathrm{e}} H_{\omega}^{(n, m)}\left[Y_{n}^{m}(\theta, \lambda) \mathrm{e}^{\mathrm{i}\left(\Theta_{\omega}(t)-\zeta_{n, m}\right)}\right. \\
& \left.+Y_{n}^{m *}(\theta, \lambda) \mathrm{e}^{-\mathrm{i}\left(\Theta_{\omega}(t)-\zeta_{n, m}\right)}\right] .
\end{aligned}
$$

The surface spherical harmonics $Y_{n}^{m},(0 \leq m \leq n)$, are defined here by

$$
\begin{aligned}
& Y_{n}^{m}(\theta, \lambda)=N_{n}^{m} P_{n}^{m}(\cos \theta) \mathrm{e}^{\mathrm{i} m \lambda} \\
& N_{n}^{m}=(-1)^{m}\left(\frac{2 n+1}{4 \pi} \frac{(n-m) !}{(n+m) !}\right)^{1 / 2} \\
& Y_{n}^{-m}=(-1)^{m} Y_{n}^{m *}, \text { and } \int Y_{n}^{m *} Y_{q}^{p} \sin \theta \mathrm{d} \theta \mathrm{d} \lambda=\delta_{n, q} \delta_{m, p}
\end{aligned}
$$


The torque on the Earth due to the potential $V_{\omega}^{(n, m)}$ is

$\boldsymbol{\Gamma}_{\omega}^{n m}=\int \rho(\boldsymbol{r})(\boldsymbol{r} \times \nabla) V_{\omega}^{(n, m)}(\boldsymbol{r} ; t) \mathrm{d}^{3} r$

where $\rho(\boldsymbol{r})$ is the density at the postion $\boldsymbol{r}$, and the integral is over the volume of the Earth. The operator $(\boldsymbol{r} \times \nabla)$ is proportional to the angular momentum operator $\boldsymbol{L}$ of quantum mechanics. Knowing the action of $\boldsymbol{L}$ on $Y_{n}^{m}$ (see, for instance, Mathews \& Venkatesan 1976), one finds immediately that

$(\boldsymbol{r} \times \nabla)_{+} Y_{n}^{m}=\mathrm{i} c_{n}^{m} Y_{n}^{m+1}$,

$(\boldsymbol{r} \times \nabla)_{-} Y_{n}^{m}=\mathrm{i} c_{n}^{-m} Y_{n}^{m-1}$,

where $(\boldsymbol{r} \times \nabla)_{ \pm}=(\boldsymbol{r} \times \nabla)_{1} \pm \mathrm{i}(\boldsymbol{r} \times \nabla)_{2}$ and

$c_{n}^{m}=[(n-m)(n+m+1)]^{1 / 2}$.

We can now carry out the angular part of the integration in (A3) with the help of a spherical harmonic expansion of $\rho(\boldsymbol{r})$ :

$\rho(\boldsymbol{r})=\sum_{l=0}^{\infty} \sum_{k=-l}^{l} \rho_{l k}(r) Y_{l}^{k^{*}}(\theta, \lambda)$,

$\rho_{n m}(r)=\int \rho(\boldsymbol{r}) Y_{n}^{m}(\theta, \lambda) \sin \theta \mathrm{d} \theta \mathrm{d} \lambda=(-1)^{m} \rho_{n,-m}^{*}(r)$.

On introducing (A6) together with (A1) into (A3) and making use of Eqs. (A2) and (A4), we obtain the following expressions for the familiar complex combination $\tilde{\Gamma}_{\omega}^{(n, m)}$ of the first two components of $\boldsymbol{\Gamma}_{\omega}^{(n m)}$ :

$$
\begin{aligned}
\tilde{\Gamma}_{\omega}^{(n, m)}= & \mathrm{i} \frac{g_{\mathrm{e}}}{2 a_{\mathrm{e}}^{n}} H_{\omega}^{(n, m)}\left[c_{n}^{m} Q_{n, m+1} \mathrm{e}^{\mathrm{i}\left(\Theta_{\omega}(t)-\zeta_{n, m}\right)}\right. \\
& \left.-c_{n}^{-m} Q_{n, m-1}{ }^{*} \mathrm{e}^{-\mathrm{i}\left(\Theta_{\omega}(t)-\zeta_{n, m}\right)}\right]
\end{aligned}
$$

for $m>0$, and

$\tilde{\Gamma}_{\omega}^{(n 0)}=\mathrm{i} \frac{g_{\mathrm{e}}}{2 a_{\mathrm{e}}^{n}} H_{\omega}^{(n, m)} c_{n}^{0} Q_{n, 1}\left[\mathrm{e}^{\mathrm{i}\left(\Theta_{\omega}(t)-\zeta_{n, 0}\right)}+\mathrm{e}^{-\mathrm{i}\left(\Theta_{\omega}(t)-\zeta_{n, 0}\right)}\right]$

for $m=0$, where

$$
Q_{n m}=\int \rho_{n m}(r) r^{n+2} \mathrm{~d} r=(-1)^{m} Q_{n,-m}^{*}
$$

The $Q_{n m}$ are related, of course, to the geopotential coefficients $\left(C_{n m}, S_{n m}\right)$. The relation is

$$
\left(2-\delta_{m 0}\right) 4 \pi N_{n m} Q_{n m}=(2 n+1) M_{\mathrm{E}} a_{\mathrm{e}}^{n}\left(C_{n m}+\mathrm{i} S_{n m}\right) .
$$

The expressions given in Eqs. (26) of the text are obtained on substituting for $Q_{n m}$ in (A8) and (A9) from (A11). The entries in Table 1 regarding the origins of the wobbles and nutations in various frequency bands are based on Eqs. (A8) and (A9). The prograde/retrograde nature of the two terms in each of these equations is evident from the fact that the contribution to $\left(\Gamma_{x}^{(n, m)}, \Gamma_{y}^{(n, m)}\right)$ from the first term is proportional to $\left(\cos \Theta_{\omega}(t), \sin \Theta_{\omega}(t)\right)$ which rotates in the prograde sense, while the contribution from the second term, proportional to $\left(\cos \Theta_{\omega}(t),-\sin \Theta_{\omega}(t)\right)$, is retrograde.

\section{References}

Bizouard, Ch., Folgueira, M., \& Souchay, J. 2001, in Proc. Journées Systèmes de Référence 2000, ed. N. Capitaine, 260

Bretagnon, P., Francou, G., Rocher, P., \& Simon, J.-L. 1998, A\&A, 329, 329

Bretagnon, P., Rocher, P., \& Simon, J.-L. 1997, A\&A, 319, 305

Brzeziński, A., \& Capitaine, N. 1993, J. Geophys. Res., 98, 6667

Brzeziński, A., \& Capitaine, N. 2002, in Proc. Journées Systèmes de Référence 2001, ed. N. Capitaine, 51

Cartwright, D. E., \& Tayler, R. J. 1971, Geophys. J. R. Astron. Soc., 23, 45

Chao, B. F., Ray, R. D., Gipson, J. M., Egbert, G. D., \& Ma, C. 1996, J. Geophys. Res., 101, 20151

Defraigne, P., Dehant, V., \& Wahr, J. M. 1996, Geophys. J. Int., 125, 173

Escapa, A., Getino, J., \& Ferrandiz, J. M. 2002, in Proc. Journées Systèmes de Référence 2001, ed. N. Capitaine, 275

Folgueira, M., Souchay, J., \& Kinoshita, H. 1998a, Celestial Mech. Dyn. Astron., 69, 373

Folgueira, M., Souchay, J., \& Kinoshita, H. 1998b, Celestial Mech. Dyn. Astron., 70, 147

Folgueira, M., Bizouard, Ch., \& Souchay, J. 2001, Celestial Mech. Dyn. Astron., 81, 191

Getino, J., Ferrandiz, J. M., \& Escapa, A. 2001, A\&A, 370, 330

Gross, R. S. 1992, Geophys. J. Int., 109, 162

Herring, T. A., Mathews, P. M., \& Buffett, B. A. 2002, J. Geophys. Res., 107, B4, 10.1029/2001JB000165, ETG 4-1

Mathews, P. M., \& Bretagnon, P. 2002, in Proc. Journées Systèmes de Référence 2001, ed. N. Capitaine, 28

Mathews, P. M., Buffett, B. A., Herring, T. A., \& Shapiro, I. I. 1991, J. Geophys. Res., 96, 8219

Mathews, P. M., Herring, T. A., \& Buffett, B. A. 2002, J. Geophys. Res., 107, B4, 10.1029/2001JB000390, ETG 3-1

Mathews, P. M., \& Venkatesan, K. 1976, A textbook of quantum mechanics, (New Delhi: Tata McGraw-Hill)

McCarthy, D. D., (ed.) 1992, IERS Standards 1992, IERS Technical Note 13, Observatoire de Paris

Morelli, A., \& Dziewonski, A. M. 1987, Nature, 325, 678

Roosbeek, F. 1996, Geophys. J. Int., 126, 197

Roosbeek, F., \& Dehant, V. 1998, Celestial Mech., 70, 215

Sailor, R. V., \& Dziewonski, A. M. 1978, Geophys. J. R. Astron. Soc., 53, 559

Sasao, T., Okubo, S., \& Saito, M. 1980, in Proc. IAU Symp. 78, ed. E. P. Federov, M. L. Smith, \& P. L. Bender (D. Reidel, Norwell, Mass.), 165

Seidelmann, P. K. 1982, 1980 IAU theory of nutation: The final report of the IAU working group on nutation, Celestial Mech., 27, 79

Souchay, J., \& Kinoshita, H. 1996, A\&A, 312, 1017

Souchay, J., \& Kinoshita, H. 1997, A\&A, 318, 639

Souchay, J., Loysel, B., Kinoshita, H., \& Folgueira, M. 1999, A\&AS, 135,111

Wahr, J. M., \& Bergen, Z. 1986, Geophys. J. R. Astron. Soc., 87, 633 